\title{
LINEAR PROGRAMMING BOUNDS FOR CODES IN GRASSMANNIAN SPACES
}

\author{
CHRISTINE BACHOC
}

\begin{abstract}
We develop the linear programming method in order to obtain bounds for the cardinality of Grassmannian codes endowed with the chordal distance. We obtain a bound and its asymptotic version that generalize the well-known bound for codes in the real projective space obtained by Kabatyanskiy and Levenshtein, and improve the Hamming bound for sufficiently large minimal distances.
\end{abstract}

\section{INTRODUCTION}

Philippe Delsarte has introduced the so-called linear programming method, in order to find bounds for the size of codes with prescribed minimal distance, in the classical case of codes over finite fields. This method, also called Delsarte method or polynomial method, exploits a certain family of orthogonal polynomials attached to the situation, the Krawtchouk polynomials, and their positivity property. These polynomials and their properties are intimately related to the action of the symmetric group on the Hamming space. Delsarte method has proved to be very powerful, and was extended to many other situations, where the underlying space is symmetric of rank one, and is homogeneous under the action of a certain group of transformations. Examples of such spaces are: the Johnson space, the Grassmannian space over a finite field, the unit sphere of the Euclidean space, the projective spaces over the real, complex and quaternionic fields (for these last spaces see [1], 2]).

In recent years, codes over the real Grassmannian space have attracted attention, motivated by their application to information theory, more precisely to the so-called space-time codes, used for multi-antenna systems of communication. The distance usually considered is the chordal distance, introduced in 3], and defined in the following way (more details are given in the next subsection): The Grassmannian space of $m$-dimensional subspaces of $\mathbb{R}^{n}$, where $m \leq n / 2$, is denoted by $\mathcal{G}_{m, n}$; to a pair $(p, q)$ of elements of $\mathcal{G}_{m, n}$ is associated $m$ principal angles $\theta_{1}, \ldots, \theta_{m} \in[0, \pi / 2]$. Let $y_{i}:=\cos ^{2} \theta_{i}$.

C. Bachoc is with the Laboratoire A2X, Institut de Mathématiques de Bordeaux, 351 cours de la Libération, 33405 Talence, France, bachoc@math.u-bordeaux1.fr. 
Then

$$
d_{c}(p, q):=\sqrt{\sum_{i=1}^{m} \sin ^{2} \theta_{i}}=\sqrt{m-\sum_{i=1}^{m} y_{i}} .
$$

In [3], the authors give bounds for the size of Grassmannian codes, called the simplex and orthoplex bounds. The main drawback of these bounds is that they are only valid in a certain range of minimal distances. In 4], an asymptotic bound, derived from the Hamming bound, is given. Another approach is developed in [5], where bounds are given for codes whose principal angles are subject to certain constraints (the so-called $f$-codes), which arise naturally from the notion of Grassmannian designs introduced in [6].

In this paper, we extend Delsarte method to the Grassmannian codes, exploiting the zonal polynomials attached to $\mathcal{G}_{m, n}$. These are symmetric polynomials in the $m$ variables $y_{1}, \ldots, y_{m}$; they belong to the family of orthogonal generalized Jacobi polynomials (see the next subsection). In the second section, we recall, or settle the properties of these polynomials needed to perform linear programming bounds; these properties are easy to obtain by straightforward generalization of the arguments used in the classical cases. In fact, the principles underlying the LP method would remain true for the zonal polynomials attached to any symmetric space. The real difficulties start when one wants to actually perform explicit bounds, because the polynomials have (for $m \geq 2$ ) several variables. The low degree cases are still easy to manage; this is done in section 3. where we recover the simplex bound as the bound arising from the case of degree one, and give new bounds from polynomials of degree 2 and 3 . In the forth section, we propose a strategy based on the eigenvalues of certain symmetric endomorphisms, which extends the one variable method based on the zeros of the polynomials and on Christoffel-Darboux formula, but avoids to deal with zeros of polynomials in several variables. We obtain an upper bound for the size of a code $C$ with minimal distance $\delta$, which is expressed in terms of the largest eigenvalue (Theorem 4.4 and Corollary 9). Section 5 settles the asymptotic behavior of this largest eigenvalue (Theorem 5.3), and in section 6] we derive the following asymptotic version of the bound:

Theorem 1.1. Let $C$ be a code in $\mathcal{G}_{m, n}$ with minimal chordal distance $\delta$, let $\left.s:=m-\delta^{2} \in\right] 0, m[$ and let

$$
\rho:=\frac{m}{2}\left(-1+\left(1-\frac{s}{m}\right)^{-1 / 2}\right) .
$$

Then, when $n \rightarrow+\infty$,

$$
\frac{1}{n} \log |C| \lesssim m((1+\rho) \log (1+\rho)-\rho \log (\rho)) .
$$

Our bound coincides with the bound given by G. Kabatiansky and V. Levenshtein in [7] for the case of the real projective space, corresponding 
to $m=1$. But it beats the Hamming bound of [4] only when the minimal distance is relatively big.

1.1. Basic facts about Grassmannian spaces and their zonal polynomials. We repeat here, without proofs, some well-known facts about Grassmannian spaces and their zonal polynomials. Some useful references for the mathematical background are: [8, 9] for the representations of the orthogonal group, 14], 15, for the Grassmannian spaces and harmonic analysis on it, 10, [11, [12, 13 for multivariate orthogonal polynomials.

The real Grassmannian space, denoted by $\mathcal{G}_{m, n}(m \leq n / 2)$, is the set of $m$-dimensional $\mathbb{R}$-linear subspaces of $\mathbb{R}^{n}$. The orthogonal group $\mathrm{O}(n, \mathbb{R})$ acts transitively on $\mathcal{G}_{m, n}$; a transformation stabilizing a given element $p_{0}$ also stabilizes its orthogonal complement $p_{0}^{\perp}$ and therefore the stabilizer of $p_{0}$ is isomorphic to the direct product $\mathrm{O}(m, \mathbb{R}) \times \mathrm{O}(n-m, \mathbb{R})$. Hence we derive the identification of $\mathcal{G}_{m, n}$ with the set of classes:

$$
\mathcal{G}_{m, n} \simeq \mathrm{O}(n, \mathbb{R}) /(\mathrm{O}(m, \mathbb{R}) \times \mathrm{O}(n-m, \mathbb{R}))
$$

from which $\mathcal{G}_{m, n}$ inherits the structure of a (compact) differential variety, and a $\mathrm{O}(n, \mathbb{R})$-invariant measure that will be normalized so that $\int_{\mathcal{G}_{m, n}} d p=$ 1. It is worth noticing that the case $m=1$ corresponds to the real projective space.

In order to understand the action of $\mathrm{O}(n, \mathbb{R})$ on pairs $(p, q) \in \mathcal{G}_{m, n}{ }^{2}$, we need to introduce the principal angles between $p$ and $q$. These are $m$ angles $\theta_{1}, \ldots, \theta_{m} \in[0, \pi / 2]$ defined in the following way:

Let $p_{1} \subset p, q_{1} \subset q$ be two lines such that the angle $\theta_{1}$ between $p_{1}$ and $q_{1}$ is minimal. If $m=1$ we have finished, otherwise let $p^{\prime}$ be the orthogonal complement of $p_{1}$ in $p, q^{\prime}$ be the orthogonal complement of $q_{1}$ in $q$; we define recursively $\theta_{2}, \ldots, \theta_{m}$ to be the principal angles associated to the pair $\left(p^{\prime}, q^{\prime}\right)$ in $\mathcal{G}_{m-1, n}$. We introduce the notation $y_{i}:=\cos ^{2} \theta_{i}$; when needed, we may denote rather $y_{i}(p, q), \theta_{i}(p, q)$. A classical result on the geometry of $\mathcal{G}_{m, n}$ is the following:

Proposition 1.2. Two pairs $(p, q)$ and $\left(p^{\prime}, q^{\prime}\right)$ are in the same orbit under the action of the group $\mathrm{O}(n, \mathbb{R})$, i.e. there exists $\sigma \in \mathrm{O}(n, \mathbb{R})$ such that $\sigma(p)=p^{\prime}$ and $\sigma(q)=q^{\prime}$, if and only if

$$
y_{i}(p, q)=y_{i}\left(p^{\prime}, q^{\prime}\right) \text { for all } 1 \leq i \leq m .
$$

The previous proposition expresses the fact that the orbits under the action of $\mathrm{O}(n, \mathbb{R})$ of the pairs $(p, q) \in \mathcal{G}_{m, n}{ }^{2}$ are characterized by the $m$ tuple of real numbers $\left(y_{1}(p, q), \ldots, y_{m}(p, q)\right)$. It becomes clear that, for $m \geq 2, \mathcal{G}_{m, n}$ is not 2-point homogeneous, i.e. a single distance on $\mathcal{G}_{m, n}$ cannot characterize these orbits (while it is the case for other spaces of interest in coding theory, like the Hamming and binary Johnson spaces, or the unit sphere of the Euclidean space). It is the reason why we shall deal with mutivariate polynomials. Also, it shows that the choice of a distance 
on $\mathcal{G}_{m, n}$ is sort of arbitrary. We shall stick to the chordal distance in this paper, as introduced in [3]:

$$
d_{c}(p, q):=\sqrt{\sum_{i=1}^{m} \sin ^{2} \theta_{i}}=\sqrt{m-\sum_{i=1}^{m} y_{i}} .
$$

Other possibilities are the Riemannian distance $\sqrt{\sum_{i=1}^{m} \theta_{i}^{2}}$ which behaves somewhat badly because it is not smooth; the max distance $\max _{i} \theta_{i}$, etc.. The "product distance" (which is not a distance in the metric sense) $\left(\prod_{i} \sin \theta_{i}\right)$ seems to be relevant in the context of space time codes.

Now we consider the space $L^{2}\left(\mathcal{G}_{m, n}\right)$ of functions $f: \mathcal{G}_{m, n} \rightarrow \mathbb{C}$ such that $\int_{\mathcal{G}_{m, n}}|f|^{2} d p<+\infty$. This is a $\mathbb{C}$-vector space, endowed with the hermitian product:

$$
<f, g>=\int_{\mathcal{G}_{m, n}} f(p) \overline{g(p)} d p
$$

and with the left action of the orthogonal group given by:

$$
(\sigma \cdot f)(p)=f\left(\sigma^{-1}(p)\right)
$$

(for which the above hermitian product is of course invariant).

Its associated algebra of zonal functions (also called the Hecke algebra) is:

$$
\begin{array}{r}
\mathcal{Z}:=\left\{Z: \mathcal{G}_{m, n}{ }^{2} \rightarrow \mathbb{C} \mid Z(p, \cdot), Z(\cdot, q) \in L^{2}\left(\mathcal{G}_{m, n}\right)\right. \text { and } \\
Z(\sigma(p), \sigma(q))=Z(p, q) \text { for all } \sigma \in \mathrm{O}(n, \mathbb{R})\}
\end{array}
$$

Form Proposition [1.2 since $Z \in \mathcal{Z}$ is constant on the orbits of $\mathrm{O}(n, \mathbb{R})$ on $\mathcal{G}_{m, n}{ }^{2}$, it can be given the form: $Z(p, q)=z\left(y_{1}(p, q), \ldots, y_{m}(p, q)\right)$ for some function $z$.

The explicit decomposition into $\mathrm{O}(n, \mathbb{R})$-irreducible subspaces of $L^{2}\left(\mathcal{G}_{m, n}\right)$, and the corresponding structure of $\mathcal{Z}$, where investigated for the first time by James and Constantine ([14]). It is now a standard result on the representation of the classical groups (see [9]).

Recall that the irreducible representations of $\mathrm{O}(n, \mathbb{R})$ are (up to a power of the determinant) naturally indexed by partitions $\kappa=\left(\kappa_{1}, \ldots, \kappa_{n}\right)$, where $\kappa_{1} \geq \cdots \geq \kappa_{n} \geq 0$ (we may omit the last parts if they are equal to 0 ). Following 9, let them be denoted by $V_{n}^{\kappa}$. For example, $V_{n}^{()}=\mathbb{C} \mathbf{1}$, and $V_{n}^{(k)}=$ Harm $_{k}$ the space of homogeneous of degree $k$, harmonic polynomials in $n$ variables.

The length $\ell(\kappa)$ of a partition $\kappa$ is the number of its non zero parts, and its degree $\operatorname{deg}(\kappa)$ also denoted by $|\kappa|$ equals $\sum_{i=1}^{n} \kappa_{i}$.

Then, the decomposition of $L^{2}\left(\mathcal{G}_{m, n}\right)$ is as follows:

$$
L^{2}\left(\mathcal{G}_{m, n}\right) \simeq \oplus V_{n}^{2 \kappa}
$$


where $\kappa$ runs over the partitions of length at most $m$ and $2 \kappa$ stands for $\left(2 \kappa_{1}, \ldots, 2 \kappa_{m}\right)$, meaning that only partitions with even parts enter the decomposition. We can see that the multiplicities in this decomposition are all equal to one, which translates the fact that the space $\mathcal{G}_{m, n}$ is a symmetric space. Consequently, to each irreducible component $V_{n}^{2 \kappa}$ is associated a uniquely determined (up to a normalizing factor) zonal function $P_{\kappa}\left(y_{1}, \ldots, y_{m}\right)$, in the sense that

$$
p(\operatorname{resp} . q) \mapsto P_{\kappa}\left(y_{1}(p, q), \ldots, y_{m}(p, q)\right) \in V_{n}^{2 \kappa}
$$

and

$$
\mathcal{Z}=\oplus_{\kappa, \ell(\kappa) \leq m} \mathbb{C} P_{\kappa} .
$$

It turns out that the $P_{\kappa}$ are symmetric polynomials in the $m$ variables $y_{1}, \ldots, y_{m}$, of degree $|\kappa|$, with rational coefficients once they are normalized by the condition $P_{\kappa}(1, \ldots, 1)=1$. Moreover, the set $\left(P_{\kappa}\right)_{|\kappa| \leq k}$ is a basis of the space of symmetric polynomials in the variables $y_{1}, \ldots, y_{m}$ of degree at most equal to $k$, denoted by $S_{k}$.

Since the irreducible subspaces of $L^{2}\left(\mathcal{G}_{m, n}\right)$ are pairwise non isomorphic, they are orthogonal for the $\mathrm{O}(n, \mathbb{R})$-invariant hermitian product defined above. This hermitian product induces an hermitian product on the space of symmetric polynomials, denoted by [, ], for which the polynomials $P_{\kappa}$ are orthogonal. More precisely, it is given by the positive measure, calculated in 14 ,

$$
d \mu=\lambda \prod_{\substack{i, j=1 \\ i<j}}^{m}\left|y_{i}-y_{j}\right| \prod_{i=1}^{m} y_{i}^{-1 / 2}\left(1-y_{i}\right)^{n / 2-m-1 / 2} d y_{i}
$$

(where $\lambda$ is chosen so that $\int_{[0,1]^{m}} d \mu(y)=1$ ). and

$$
[f, g]=\int_{[0,1]^{m}} f(y) \overline{g(y)} d \mu(y) .
$$

One recognizes a special case of the orthogonal measure associated to generalized Jacobi polynomials (11]).

We let $\Pi_{k}$ be the subspace of $S_{k}$ generated by the polynomials $P_{\kappa}$, with $|\kappa|=k$, so that we have the orthogonal decomposition:

$$
S_{k}=S_{k-1} \perp \Pi_{k} .
$$

Let the dimensions of $S_{k}, \Pi_{k}$ be denoted respectively by $s_{k}, \pi_{k}$. The number $\pi_{k}$ is also equal to the number of partitions $\kappa$ of $k$ in at most $m$ parts. These dimensions also depend on $m$, although it does not reflect on our notation, for the sake of simplicity.

In view of the explicit calculation of the polynomials $P_{\kappa}$, it is better to use the following characterization, which involves the polynomials $C_{\kappa}$, which are themselves the zonal polynomials associated to the symmetric space $\mathrm{GL}(m, \mathbb{R}) / \mathrm{O}(m, \mathbb{R})$ (these polynomials are Jack polynomials, normalized by 
$C_{\kappa}(1, \ldots, 1)=1$, see [14, 12]), and the differential operator $\Delta$ induced on $\mathbb{C}\left[y_{1}, \ldots, y_{m}\right]^{S_{m}}$ by the Laplace Beltrami operator of $\mathcal{G}_{m, n}$. The condition: for all $1 \leq i \leq m, \kappa_{i} \geq \mu_{i}$ is denoted by: $\kappa \geq \mu$.

(i) $P_{\kappa}$ is an eigenvector for the operator

$$
\begin{aligned}
\Delta:= & \sum_{i=1}^{m} y_{i}^{2} \frac{\partial^{2}}{\partial y_{i}^{2}}+\sum_{i \neq j=1}^{m} y_{i}^{2}\left(y_{i}-y_{j}\right)^{-1} \frac{\partial}{\partial y_{i}} \\
& +\left(\frac{n}{2}-m+1\right) \sum_{i=1}^{m} y_{i} \frac{\partial}{\partial y_{i}}-\sum_{i=1}^{m} y_{i} \frac{\partial^{2}}{\partial y_{i}^{2}} \\
& -\sum_{i \neq j=1}^{m} y_{i}\left(y_{i}-y_{j}\right)^{-1} \frac{\partial}{\partial y_{i}}-\frac{1}{2} \sum_{i=1}^{m} \frac{\partial}{\partial y_{i}}
\end{aligned}
$$

(ii) $P_{\kappa}=\beta_{\kappa} C_{\kappa}+\sum_{\mu \mid \kappa>\mu} \beta_{\kappa, \mu} C_{\mu}$

(iii) $P_{\kappa}(1, \ldots, 1)=1$.

Condition (ii) is needed to avoid the multiplicities of the operator $\Delta$.

Examples: the effective computation of the polynomials $P_{\kappa}$ following the method described above leads to, up to the normalization imposed by (iii):

$$
\begin{aligned}
& P_{0}=1 \\
& P_{(1)}=s_{1}-\frac{m^{2}}{n} \\
& P_{(11)}=\sigma_{1}-\frac{(m-1)^{2}}{n-2} s_{1}+\frac{m^{2}(m-1)^{2}}{2(n-1)(n-2)} \\
& P_{(2)}=s_{2}+\frac{2}{3} \sigma_{1}-\frac{2(m+2)^{2}}{3(n+4)} s_{1}+\frac{m^{2}(m+2)^{2}}{3(n+2)(n+4)}
\end{aligned}
$$

where $s_{1}=\sum_{1 \leq i \leq m} y_{i}, s_{2}=\sum_{1 \leq i \leq m} y_{i}^{2}$,

$\sigma_{1}=\sum_{1 \leq i<j \leq m} y_{i} y_{j}$.

Remark: The complex Grassmannian $\mathcal{G}_{m, n}(\mathbb{C})$ is more commonly used in the context of space-time coding. It affords the transitive action of the unitary group $U(\mathbb{C}, n)$; similarly one defines principal angles $\left(\theta_{1}, \ldots, \theta_{m}\right)$ between two elements of $\mathcal{G}_{m, n}(\mathbb{C})$. The $U(\mathbb{C}, n)$ decomposition of $L^{2}\left(\mathcal{G}_{m, n}(\mathbb{C})\right)$ and the associated zonal polynomials are computed in 14] so one can play the same game concerning bounds of codes. On the other hand, a bound is also obtained from the embedding $\mathcal{G}_{m, n}(\mathbb{C}) \subset \mathcal{G}_{2 m, 2 n}(\mathbb{R})$ (if $\left(\theta_{1}, \ldots, \theta_{m}\right)$ are the principal angles associated to a pair $(p, q)$ of elements in $\mathcal{G}_{m, n}(\mathbb{C})$, the $2 m$ principal angles associated to the pair $(p, q)$, seen as elements of $\mathcal{G}_{2 m, 2 n}(\mathbb{R})$, are simply $\left.\left(\theta_{1}, \theta_{1}, \theta_{2}, \theta_{2}, \ldots\right)\right)$. 


\section{Zonal polynomials associated to $\mathcal{G}_{m, n}$ AND the LP BOUnd}

In this section, we settle the properties of the polynomials $P_{\kappa}$ relevant for the LP bound, settle this bound, and show how the Christoffel-Darboux formula can be exploited in that context.

The dimension of $V_{n}^{\kappa}$ is denoted by $d_{\kappa}$. Explicit formulas for $d_{\kappa}$ can be found in [8]; however we do not need them before Section 5 .

Proposition 2.1. The polynomials $P_{\kappa}$, normalized by the condition $P_{\kappa}(1, \ldots, 1)=1$, satisfy:

(i) $\left[P_{\kappa}, P_{\kappa}\right]=d_{2 \kappa}^{-1}$

(ii) (Positivity property): For all finite set $C \subset \mathcal{G}_{m, n}$,

$$
\sum_{p, q \in C} P_{\kappa}\left(y_{1}(p, q), \ldots, y_{m}(p, q)\right) \geq 0
$$

(iii) Let $p_{\kappa, \mu}^{\nu}$ be defined by the property:

$$
P_{\kappa} P_{\mu}=\sum_{\nu} p_{\kappa, \mu}^{\nu} P_{\nu}
$$

The numbers $p_{\kappa, \mu}^{\nu}$ are non-negative numbers.

Proof. These properties where already pointed out in [5] [Lemma 2.2] and step on very general arguments (see [16] [Theorem 3.1]). For the sake of completeness, we briefly recall the arguments. Let $e_{1}, \ldots, e_{d_{2 \kappa}}$ be any orthonormal basis of the subspace $\mathrm{H}_{m, n}^{2 \kappa}$ of $L^{2}\left(\mathcal{G}_{m, n}\right)$ isomorphic to $V_{n}^{2 \kappa}$. Let $\tilde{P}_{\kappa}(p, q):=P_{\kappa}\left(y_{1}(p, q), \ldots, y_{m}(p, q)\right)$. It is well known that we have (this is called the addition formula)

$$
\tilde{P}_{\kappa}(p, q)=\frac{1}{d_{2 \kappa}} \sum_{i=1}^{d_{2 \kappa}} e_{i}(p) \overline{e_{i}(q)} .
$$

As a consequence, from the expression

$$
\left[P_{\kappa}, P_{\kappa}\right]=\int_{\mathcal{G}_{m, n}} \tilde{P}_{\kappa}(p, q) \tilde{P}_{\kappa}(q, p) d q
$$

(i) follows. Moreover,

$$
\begin{aligned}
\sum_{p, q \in C} \tilde{P}_{\kappa}(p, q) & =\frac{1}{d_{2 \kappa}} \sum_{p, q \in C}\left(\sum_{i=1}^{d_{2 \kappa}} e_{i}(p) \overline{e_{i}(q)}\right) \\
& =\frac{1}{d_{2 \kappa}} \sum_{i=1}^{d_{2 \kappa}}\left(\sum_{p, q \in C} e_{i}(p) \overline{e_{i}(q)}\right) \\
& =\frac{1}{d_{2 \kappa}} \sum_{i=1}^{d_{2 \kappa}}\left|\sum_{p \in C} e_{i}(p)\right|^{2} \geq 0 .
\end{aligned}
$$


hence (ii). More generally, for any function $\alpha: C \rightarrow \mathbb{C}$, we have:

$$
\begin{aligned}
\sum_{p, q \in C} \alpha(p) \overline{\alpha(q)} \tilde{P}_{\kappa}(p, q) & =\frac{1}{d_{2 \kappa}} \sum_{i=1}^{d_{2 \kappa}}\left|\sum_{p \in C} \alpha(p) e_{i}(p)\right|^{2} \\
& \geq 0 .
\end{aligned}
$$

Conversely, assume $F \in S_{k}$ is a polynomial with real coefficients, such that, for any finite set $C \subset \mathcal{G}_{m, n}$ and any function $\alpha: C \rightarrow \mathbb{C}$,

$$
\sum_{p, q \in C} \alpha(p) \overline{\alpha(q)} \tilde{F}(p, q) \geq 0
$$

and let us prove that $F$ expands on the $P_{\kappa}$ with non-negative coefficients. Taking limits, we have, for any $\alpha \in L^{2}\left(\mathcal{G}_{m, n}\right)$,

$$
\iint_{\mathcal{G}_{m, n}} \alpha(p) \overline{\alpha(q)} \tilde{F}(p, q) d p d q \geq 0
$$

and hence, using the addition formula,

$$
\iint_{\mathcal{G}_{m, n}} \tilde{P}_{\kappa}(p, q) \tilde{F}(p, q) d p d q \geq 0 .
$$

If $F=\sum_{|\nu| \leq k} f_{\nu} P_{\nu}$, the left hand-side equals $f_{\kappa} / d_{2 \kappa}$, which proves that the coefficients $f_{\kappa}$ are non-negative numbers. Using once again the addition formula, it is easy to show that the product $P_{\kappa} P_{\mu}$ holds this general positivity property, and therefore expands on the $P_{\nu}$ with non-negative coefficients.

2.1. The principles of the LP bound. The positivity property of the polynomials $P_{\kappa}$ is the basis of the linear programming method to upper bound the cardinality of $\delta$-codes.

Definition 2.2. A Grassmannian code $C$ satisfying the constraint:

$$
\text { For all } p \neq q \in C^{2}, d_{c}(p, q) \geq \delta \text {. }
$$

is called a $\delta$-code.

Proposition 2.3. Assume $F_{k} \in S_{k}$ satisfy:

(i) $F_{k}=\sum_{|\kappa| \leq k} f_{\kappa} P_{\kappa}$ with $f_{\kappa} \geq 0$ for all $\kappa, f_{0}>0$

(ii) $F_{k}\left(y_{1}, \ldots, y_{m}\right) \leq 0$ for all $\left(y_{1}, \ldots, y_{m}\right) \in[0,1]^{m}$ such that $\sum_{i=1}^{m} y_{i} \leq m-\delta^{2}$

Then, the following bound holds for the cardinality $|C|$ of any $\delta$-code:

$$
|C| \leq \frac{F_{k}(1, \ldots, 1)}{f_{0}} .
$$


Proof. This is a standard argument, that we recall here. Let $C$ be a $\delta$-code. As before, we let $\tilde{F}_{k}(p, q)=F_{k}\left(y_{1}(p, q), \ldots, y_{m}(p, q)\right)$. We calculate

$$
\sum_{p, q \in C} \tilde{F}_{k}(p, q)=\sum_{|\kappa| \leq k} f_{\kappa}\left(\sum_{p, q \in C} \tilde{P}_{\kappa}(p, q)\right)
$$

Assumption (ii) leads to $\tilde{F}_{k}(p, q) \leq 0$ when $p \neq q$. The remaining terms of the left hand-side, corresponding to $p=q$, contribute by $|C| F_{k}(1, \ldots, 1)$. Assumption (i), together with the positivity property of the polynomials $P_{\kappa}$ (Proposition 2.1 (ii)), show that all the terms of the right hand-side are non-negative. When $\kappa=(0), P_{\kappa}=1$ and the contribution is $f_{0}|C|^{2}$. We obtain

$$
|C| F_{k}(1, \ldots, 1) \geq f_{0}|C|^{2}
$$

equivalently

$$
|C| \leq \frac{F_{k}(1, \ldots, 1)}{f_{0}}
$$

It is worth noticing that equality in this inequality happens if and only if, for all $1 \leq|\kappa| \leq k$ such that $f_{\kappa} \neq 0, \sum_{p, q \in C} \tilde{P}_{\kappa}(p, q)=0$ and, for all $p \neq q \in C$, $\tilde{F}_{k}(p, q)=0$. The first condition says that $C$ is a $2 k$-design in the sense of $[6]$ (when it holds for all $1 \leq|\kappa| \leq k$ ), and the second one that $C$ is an $F_{k}$-code in the sense of [5].

\subsection{The three-term relation and the Christoffel-Darboux formula.}

We join here more material on the sequence of polynomials $P_{\kappa}$, that will be of later use. The results presented here are essentially established in [13, except that we deal with symmetric polynomials. Following [13], the (column) vector of the polynomials $P_{\kappa}$ with $|\kappa|=k$ is denoted by $\mathbb{P}_{k}$. If necessary, we order the partitions of the same degree in increasing lexicographic order.

We also set

$$
\sigma:=y_{1}+y_{2}+\cdots+y_{m}
$$

and, when necessary, we make the involved variables explicit, by writing $\sigma(y)$ rather than $\sigma$. The $\pi_{k} \times \pi_{k}$ diagonal matrix, denoted by $D_{k}$, with entries

$$
D_{k}[\kappa, \kappa]=d_{2 \kappa}:=\operatorname{dim}\left(V_{n}^{2 \kappa}\right)
$$

is the inverse of the Gram matrix of $\mathbb{P}_{k}$.

Next result is an analogue of the so-called "three-term relation".

Theorem 2.4. For all $k \geq 1$, there exists matrices $A_{k}, B_{k}, C_{k}$, of size respectively $\pi_{k} \times \pi_{k+1}, \pi_{k} \times \pi_{k}, \pi_{k} \times \pi_{k-1}$, such that:

$$
\sigma \mathbb{P}_{k}=A_{k} \mathbb{P}_{k+1}+B_{k} \mathbb{P}_{k}+C_{k} \mathbb{P}_{k-1} \text {. }
$$

Moreover, $\left(D_{k} B_{k}\right)^{t}=D_{k} B_{k}$ and $D_{k} C_{k}=\left(D_{k-1} A_{k-1}\right)^{t}$. 
Proof. The polynomials $\sigma P_{\kappa}$ with $|\kappa|=k$ are symmetric of total degree $k+1$ so they afford a decomposition over the $\left(P_{\mu}\right)_{|\mu| \leq k+1}$. Moreover, $\left[\sigma P_{\kappa}, P_{\mu}\right]=$ $\left[P_{\kappa}, \sigma P_{\mu}\right]=0$ if $|\mu| \leq k-2$.

If $|\mu|=|\kappa|=k$, we have: $B_{k}[\kappa, \mu]\left[P_{\mu}, P_{\mu}\right]=\left[\sigma P_{\kappa}, P_{\mu}\right]=\left[P_{\kappa}, \sigma P_{\mu}\right]=$ $B_{k}[\mu, \kappa]\left[P_{\kappa}, P_{\kappa}\right]$, which proves that the matrix $D_{k} B_{k}$ is symmetric. The same argument shows that $D_{k} C_{k}=\left(D_{k-1} A_{k-1}\right)^{t}$.

Notations: We want to define $\kappa^{(i)}$ (respectively $\kappa_{(i)}$ ) to be the partition obtained from $\kappa$ by increasing (respectively decreasing) the $i$-th part $\kappa_{i}$ by one. This is not possible for all $i$, since the result should be also a partition, i.e. the new parts should be in decreasing order. Hence we define (where $\left.\kappa_{m+1}:=0\right)$ :

$$
\left\{\begin{array}{l}
u(\kappa):=\{1\} \cup\left\{i \in[2, m] \mid \kappa_{i-1}>\kappa_{i}\right\} \\
d(\kappa):=\left\{i \in[1, m] \mid \kappa_{i}>\kappa_{i+1}\right\}
\end{array}\right.
$$

The set $u(\kappa)$ is the set of indices $i$ for which $\kappa^{(i)}$ makes sense (respectively $d(\kappa)$ for $\left.\kappa_{(i)}\right)$. Moreover, if $|\kappa|=k,\left|\kappa^{(i)}\right|=k+1$ and $\left|\kappa_{(i)}\right|=k-1$.

Otherwise explicitly mentioned, in the rest of this paper, $\kappa, \kappa^{\prime}$ are partitions of degree $k$, while $\mu, \mu^{\prime}$ are partitions of degree $k+1$ and $\nu, \nu^{\prime}$ are partitions of degree $k-1$.

Proposition 2.5. The following properties hold:

(i) For all $\kappa, \mu$ and $\kappa^{\prime}, A_{k}[\kappa, \mu] \geq 0$, and $B_{k}\left[\kappa, \kappa^{\prime}\right] \geq 0$.

(ii) The coefficients of the matrix $A_{k}$ are equal to zero, except the coefficients $A_{k}\left[\kappa, \kappa^{(i)}\right]$, which are positive.

Proof. The first assertions are equivalent to: $\left[\sigma P_{\kappa}, P_{\mu}\right] \geq 0$ for all $\kappa, \mu$ of any degree. But $\left[\sigma P_{\kappa}, P_{\mu}\right]=\left[1, \sigma P_{\kappa} P_{\mu}\right]$ and $\sigma=m\left(1-\frac{m}{n}\right) P_{1}+\frac{m^{2}}{n}$. Joint with Proposition 2.1(iii), we obtain $\left[1, \sigma P_{\kappa} P_{\mu}\right] \geq 0$.

The coefficients $A_{k}[\kappa, \mu]$ can be more precisely calculated, using ([17, Lemma 7.5.7]). Since we do not normalize the polynomials $C_{\kappa}$ in the same way, we introduce coefficients $\left[\begin{array}{l}\mu \\ \kappa\end{array}\right]$ such that

$$
\sigma C_{\kappa}=\sum_{|\mu|=k+1}\left[\begin{array}{l}
\mu \\
\kappa
\end{array}\right] C_{\mu} .
$$

They differ by a positive multiplicative factor from the generalized binomial coefficients $\left(\begin{array}{l}\mu \\ \kappa\end{array}\right)$ defined in [17]; see also [10]. Then we have

$$
A_{k}[\kappa, \mu]=\left[\begin{array}{l}
\mu \\
\kappa
\end{array}\right]\left(\frac{\beta_{\mu}}{\beta_{\kappa}}\right)^{-1}
$$

It is known that the generalized binomial coefficients $\left(\begin{array}{l}\mu \\ \kappa\end{array}\right)$ are equal to zero when $\mu$ is not equal to one of the $\kappa^{(i)}$; consequently the same holds for $A_{k}[\kappa, \mu]$. Moreover, since $\left(\begin{array}{c}\kappa^{(i)} \\ \kappa\end{array}\right)>0$, also $\left[\begin{array}{c}\kappa^{(i)} \\ \kappa\end{array}\right]>0$ and $A_{k}\left[\kappa, \kappa^{(i)}\right] \neq 0$. 
Theorem 2.6 (Christoffel-Darboux Formula). Let

$$
Q_{\kappa}:=\sum_{|\mu|=k+1} A_{k}[\kappa, \mu] P_{\mu} \in \Pi_{k+1} .
$$

With the previous notations, we have:

(i) For all $k \geq 0$,

$$
\begin{aligned}
& \sum_{|\nu| \leq k} d_{2 \nu} P_{\nu}(x) P_{\nu}(y)= \\
& \frac{\sum_{|\kappa|=k} d_{2 \kappa}\left(Q_{\kappa}(x) P_{\kappa}(y)-P_{\kappa}(x) Q_{\kappa}(y)\right)}{\sigma(x)-\sigma(y)}
\end{aligned}
$$

(ii) Moreover, if $\epsilon:=\sum_{i=1}^{m} \frac{\partial}{\partial y_{i}}$,

$$
\begin{aligned}
& \sum_{|\nu| \leq k} d_{2 \nu}\left(P_{\nu}(y)\right)^{2}= \\
& \sum_{|\kappa|=k} \frac{d_{2 \kappa}}{m}\left(\left(\epsilon Q_{\kappa}(y)\right) P_{\kappa}(y)-\left(\epsilon P_{\kappa}(y)\right) Q_{\kappa}(y)\right) .
\end{aligned}
$$

Proof. The proof of (i) is the same as [13] [Theorem 3.5.3]. Note that we cannot hope for a formula for each $x_{i}$ like in [13, since we should stick to symmetric polynomials. If

$$
\begin{aligned}
\Sigma_{s}: & =\left(A_{s} \mathbb{P}_{s+1}(x)\right)^{t} D_{s} \mathbb{P}_{s}(y)-\mathbb{P}_{s}(x)^{t} D_{s} A_{s} \mathbb{P}_{s+1}(y) \\
& =\sum_{|\kappa|=s} d_{2 \kappa}\left(Q_{\kappa}(x) P_{\kappa}(y)-P_{\kappa}(x) Q_{\kappa}(y)\right),
\end{aligned}
$$

from the "three-term relation" of Theorem 2.4, we have:

$$
\Sigma_{s}-\Sigma_{s-1}=(\sigma(x)-\sigma(y)) \mathbb{P}_{s}(x)^{t} D_{s} \mathbb{P}_{s}(y) .
$$

The formula (i) follows from summing up these identities, for $1 \leq s \leq k$.

In the equation (i), we replace $Q_{\kappa}(x) P_{\kappa}(y)-P_{\kappa}(x) Q_{\kappa}(y)$ by

$$
Q_{\kappa}(x)\left(P_{\kappa}(y)-P_{\kappa}(x)\right)-P_{\kappa}(x)\left(Q_{\kappa}(y)-Q_{\kappa}(x)\right) .
$$

Then, if we specialize $x_{2}=y_{2}, \ldots, x_{m}=y_{m}$ and let $x_{1}$ tend to $y_{1}$, we obtain

$$
\begin{aligned}
& \sum_{|\nu| \leq k} d_{2 \nu}\left(P_{\nu}(y)\right)^{2}= \\
& \sum_{\substack{|\kappa|=k \\
|\mu|=k+1}} d_{2 \kappa} A_{k}[\kappa, \mu]\left(\left(\frac{\partial Q_{\kappa}}{\partial y_{1}}(y)\right) P_{\kappa}(y)-\left(\frac{\partial P_{\kappa}}{\partial y_{1}}(y)\right) Q_{\kappa}(y)\right) .
\end{aligned}
$$


The same identity holds when one replaces $y_{1}$ by any $y_{i}$; if we sum up all these identities, we obtain the more symmetric formula (ii).

Remark 2.7. The left hand side of the Christoffel-Darboux formula

$$
K_{k}(x, y):=\sum_{|\nu| \leq k} d_{2 \nu} P_{\nu}(x) P_{\nu}(y) .
$$

is the reproducing kernel of the space of symmetric polynomials of degree at most $k$. It satisfies the characteristic property: for all $Q \in S_{k},\left[K_{k}(x,), Q.\right]=$ $Q(x)$.

2.3. An LP bound from Christoffel-Darboux formula. In the classical cases, Christoffel-Darboux formula is involved in the setting up of bounds of the type $|C| \leq M(\delta)$ where $M(\delta)$ is an explicit function of $\delta$. Usually the running interval of $\delta$ is divided into subintervals, related to the zeros of the zonal polynomials. This is the line followed in [18, and also in [7]; see 19 for a unified presentation. In this section, we follow this method, and analyze the difficulties arising from the several variables situation.

The numerator, of degree $k+1$, of the right hand side of ChristoffelDarboux formula (Theorem 2.6(i)) is denoted by $N_{k+1}(x, y)$. We consider the polynomial in the variables $y_{1}, \ldots, y_{m}$, of degree $2 k+1$,

$$
\begin{aligned}
F_{2 k+1}(x, y): & =-N_{k+1}(x, y) K_{k}(x, y) \\
& =\frac{N_{k+1}(x, y)^{2}}{\sigma(y)-\sigma(x)} .
\end{aligned}
$$

In order to make clear that only the $y_{1}, \ldots, y_{m}$ are variables, while the $x_{1}, \ldots, x_{m}$ will specialize to real values, we denote it by $F_{2 k+1}(x, \cdot)$.

Proposition 2.8. Let $x \in[0,1]^{m}$ satisfy $\sigma(x)>s:=m-\delta^{2}$. Assume the following conditions hold:

(i) For all $\kappa,|\kappa| \leq k, P_{\kappa}(x) \geq 0$

(ii) For all $\kappa,|\kappa|=k, Q_{\kappa}(x) \leq 0$

Then, $F_{2 k+1}(x, \cdot)$ satisfies the conditions required in Proposition 2.3 .

Proof. We have:

$$
F_{2 k+1}(x, y)=\frac{N_{k+1}(x, y)^{2}}{\sigma(y)-\sigma(x)}
$$

hence condition (ii) is satisfied when $s<\sigma(x)$.

To prove condition (i), we point out that, if $F$ and $G$ are two polynomials with non-negative coefficients on the $P_{\kappa}$, then the product $F G$ holds the same property. This is a direct consequence of Proposition 2.1(iii). 
From the definition of $K_{k}(x, y)$, its coefficient on $P_{\nu}$ with $|\nu| \leq k$ equals $d_{2 \nu} P_{\nu}(x)$ (and for higher degree partitions it is zero). On the other hand,

$$
\begin{aligned}
-N_{k+1}(x, y)= & \sum_{|\kappa|=k} d_{2 \kappa}\left(P_{\kappa}(x) Q_{\kappa}(y)-Q_{\kappa}(x) P_{\kappa}(y)\right) \\
= & \sum_{|\mu|=k+1}\left(\sum_{|\kappa|=k} d_{2 \kappa} A_{k}[\kappa, \mu] P_{\kappa}(x)\right) P_{\mu}(y) \\
& -\sum_{|\kappa|=k} d_{2 \kappa} Q_{\kappa}(x) P_{\kappa}(y)
\end{aligned}
$$

The coefficient $A_{k}[\kappa, \mu]$ is always non-negative. Clearly, under the conditions of the proposition, the coefficients of $-N_{k+1}(x, y)$ on the $P_{\kappa}$ and $P_{\mu}$ are non-negative.

Corollary 2.9. Assume $x$ satisfies the conditions of Proposition 2.8. Then, for all $\delta$-code $C$,

$$
|C| \leq \frac{(m-\sigma(x))\left(\sum_{|\nu| \leq k} d_{2 \nu} P_{\nu}(x)\right)^{2}}{-\sum_{|\kappa|=k} d_{2 \kappa} P_{\kappa}(x) Q_{\kappa}(x)}
$$

Proof. In order to apply Proposition 2.3. we are left with the computation of $f_{0}$ and of $F_{2 k+1}(x,(1, \ldots, 1))$. Since $F_{2 k+1}(x, y)=K_{k}(x, y)^{2}(\sigma(y)-\sigma(x))$, we have

$$
F_{2 k+1}(x,(1, \ldots, 1))=\left(\sum_{|\nu| \leq k} d_{2 \nu} P_{\nu}(x)\right)^{2}(m-\sigma(x)) .
$$

Using the orthogonality of the $P_{\kappa}$, we obtain

$$
\begin{aligned}
f_{0}: & =\left[F_{2 k+1}(x, \cdot), 1\right]=-\left[K_{k}(x, \cdot), N_{k+1}(x, \cdot)\right] \\
& =-\left[\sum_{|\nu| \leq k} d_{2 \nu} P_{\nu}(x) P_{\nu}, \sum_{|\kappa|=k} d_{2 \kappa} Q_{\kappa}(x) P_{\kappa}\right] \\
& =-\sum_{|\kappa|=k} d_{2 \kappa} P_{\kappa}(x) Q_{\kappa}(x) .
\end{aligned}
$$

The main problem with this approach, is that, in general, we don't even know if the inequalities (i) and (ii) of Proposition 2.8 have a solution $x$. In case these inequalities define a non empty area of $\mathbb{R}^{m}$, a second problem would be to optimize the choice of $x$ in this area. In the classical case $m=1$, $Q_{k}=P_{k+1}$ (up to a positive multiplicative factor). The interlacing property of the real zeros of the orthogonal polynomials $P_{k}$, ensures that one can take $x \in\left[z_{k}, z_{k+1}\right]$, where $z_{k}$ is the largest zero of $P_{k}$, so that $P_{k+1}(x) \leq 0$ and $P_{i}(x) \geq 0$ for all $i \leq k$. Moreover, one uses asymptotic estimates of these zeros to derive an asymptotic bound for the size of codes.

In the general case $m \geq 2$, we don't have such tools to deal with the inequalities of Proposition 2.8 which seem to be intractable in general. The 
first case $k=1$, leading to a polynomial of degree 3, is however discussed in the next section. On the other hand, one can think of the zeros of orthogonal polynomials in one variable as being the eigenvalues of the so-called Jacobi matrices associated to the sequence of polynomials. We study in section 4 the eigenvalues of the analogous matrices in the general case, and derive bounds for codes, which contain as a special case the bound obtained from a possible solution of these inequalities.

\section{LP BOUNDS OF SMALL DEGREE}

We take the following notations: let $s:=m-\delta^{2}$, the maximal value of $\sigma$ among pairs of points of a code $C$. We are looking for a function $M(s)$ such that $|C| \leq M(s)$. Obviously, $M(s)$ is an increasing function. In this section, we discuss the cases of small degree $k$, trying to optimize the choice of $F_{k}$ in Proposition 2.3

3.1. Degree 1. Let $F_{1}=1+f_{1} P_{1}$, with $f_{1} \geq 0$ (condition (i)). We have $P_{1}=\frac{n}{m(n-m)}\left(\sigma-\frac{m^{2}}{n}\right)$.

When $\sigma \in[0, s], 1+f_{1} P_{1}$ should be non-positive (condition (ii)). Therefore, The zero of $1+f_{1} P_{1}$ should be greater than $s$. It leads to the condition:

$$
s-\frac{m^{2}}{n} \leq-\frac{m(n-m)}{n f_{1}} .
$$

Since $f_{1} \geq 0$, we obtain the necessary condition $s \leq \frac{m^{2}}{n}$. The smallest value for $f_{1}$ is then

$$
f_{1}=\frac{-m(1-m / n)}{s-\frac{m^{2}}{n}}
$$

corresponding to a polynomial proportional to $\sigma-s$. We obtain the bound

$$
\text { if } s<\frac{m^{2}}{n},|C| \leq \frac{m-s}{\frac{m^{2}}{n}-s}
$$

which is the so-called simplex bound proved in 3 .

3.2. Degree 2. We restrict ourselves to polynomials which are divisible by $\sigma-s$. Then, such polynomials are polynomials in $\sigma$. We write:

$$
F_{2}=(\sigma-s)(\sigma-b)=f_{2} P_{2}+f_{11} P_{11}+f_{1} P_{1}+f_{0} .
$$

with the condition that $b \leq 0$. With $t=s-m^{2} / n$, we find: 


$$
\begin{aligned}
f_{2} & :=\frac{m(m+2)(n-m)(n-m+2)}{3(n+2)(n+4)} \\
f_{11} & :=\frac{2 m(m-1)(n-m)(n-m-1)}{3(n-2)(n-1)} \\
f_{1} & :=m\left(1-\frac{m}{n}\right)\left(\frac{m^{2}}{n}+\frac{4(n-2 m)^{2}}{n(n-2)(n+4)}-t-b\right) \\
f_{0} & :=\frac{2 m^{2}(n-m)^{2}}{n^{2}(n-1)(n+2)}-\frac{m^{2}}{n} t+b t
\end{aligned}
$$

The condition $f_{0}>0$, when $t>0$, is equivalent to

$$
b>\frac{m^{2}}{n}\left(1-\frac{2(n-m)^{2}}{\operatorname{tn}(n-1)(n+2)}\right)
$$

(and when $t<=0$ is always fulfilled), which implies

$$
t<\frac{2(n-m)^{2}}{n(n-1)(n+2)} .
$$

The condition $f_{1} \geq 0$ is equivalent to

$$
b \leq \frac{m^{2}}{n}+\frac{4(n-2 m)^{2}}{n(n-2)(n+4)}-t .
$$

One can check that the right hand side of (4) is positive for $m \geq 2$, when $t$ satisfies (3).

The bound $B=\left(f_{2}+f_{11}+f_{1}+f_{0}\right) / f_{0}$ equals

$$
B=\delta^{2} \frac{m-b}{f_{0}}
$$

As a function of $b$, it is decreasing when $t \in\left[\frac{-2 m(n-m)}{n(n-1)(n+2)}, \frac{2(n-m)^{2}}{n(n-1)(n+2)}[\right.$, and hence the best choice of $b$ is $b=0$. We obtain the bound:

Theorem 3.1. If $s \in] 0, \frac{m^{2}}{n}+\frac{2(n-m)^{2}}{n(n-1)(n+2)}[$,

$$
|C| \leq \frac{n}{m}\left(\frac{m-s}{-s+\frac{m^{2}}{n}+\frac{2(n-m)^{2}}{n(n-1)(n+2)}}\right)
$$

This bound, which is an increasing function of $\mathrm{s}$, improves on the simplex bound when $s \geq \frac{m^{2}}{n}-\frac{2 m(n-m)}{n(n-1)(n+2)}$. Their common value at $s=\frac{m^{2}}{n}-$ 
$\frac{2 m(n-m)}{n(n-1)(n+2)}$ is $\left(\begin{array}{c}n+1 \\ 2\end{array}\right)$. However, the orthoplex bound proved in [3, (5.6)] reads:

$$
\begin{aligned}
& s<m^{2} / n \Rightarrow|C| \leq\left(\begin{array}{c}
n+1 \\
2
\end{array}\right) \\
& s=m^{2} / n \Rightarrow|C| \leq(n-1)(n+2)
\end{aligned}
$$

and is better than (6) in the range $] \frac{m^{2}}{n}-\frac{2 m(n-m)}{n(n-1)(n+2)}, \frac{m^{2}}{n}[$. If we plug in (16) the value $s=m^{2} / n$, we find that $|C| \leq \frac{(n-1)(n+2)}{2(1-m / n)}$ which is better than the orthoplex bound when $m<n / 2$. We recall that the orthoplex bound is attained for a family of codes with $n=2^{i}, m=n / 2$, constructed in 20, Theorem 1]. These codes are also optimal 6-designs (see [21]).

3.3. Degree 3. We do not study general polynomials of degree 3 but rather apply the approach described in subsection 2.3

The polynomial $F_{3}$ has degree 3 , and is again a polynomial in $\sigma$. In the following, we calculate the best choice for $x$ (and discuss its existence). Let $u:=\sigma(x)-m^{2} / n$. We should have:

(i) $u \geq s-m^{2} / n$

(ii) $u \geq 0$ (Condition (i))

(iii) $u^{2}-\frac{4(n-2 m)^{2}}{n(n-2)(n+4)} u-\frac{2 m^{2}(n-m)^{2}}{n^{2}(n-1)(n+2)} \leq 0$ (Condition (ii))

The polynomial of degree 2 occurring in (iii) has a positive discriminant, and a unique positive root that we shall denote by $u_{2}$. Let $b$ and $c$ be the coefficients of this polynomial, so that it is equal to $u^{2}-b u-c$, and let $d:=\frac{2 m(n-m)}{n(n-1)(n+2)}$. The bound is then equal to:

$$
B(u):=-\frac{(n-1)(n+2)(u+d)^{2}\left(m-u-m^{2} / n\right)}{2 u\left(u^{2}-b u-c\right)} .
$$

The calculation of $B^{\prime}(u)$ shows that it is increasing in the range $\left[u_{1}, u_{2}\right]$ (the numerator has the form: $u+d$ times a degree 3 polynomial with a unique real root $\left.u_{1}\right)$. Hence, for $s \in\left[u_{1}+\frac{m^{2}}{n}, u_{2}+\frac{m^{2}}{n}\right]$, the best choice for $u$ is $u=s-m^{2} / n$.

We obtain:

Theorem 3.2. Let $d=\frac{2 m(n-m)}{n(n-1)(n+2)}, b=\frac{4(n-2 m)^{2}}{n(n-2)(n+4)}, c=\frac{2 m^{2}(n-m)^{2}}{n^{2}(n-1)(n+2)}$.

$$
\begin{aligned}
& \text { If } s \in] \frac{m^{2}}{n}, \frac{m^{2}}{n}+\frac{b}{2}+\sqrt{\frac{b^{2}}{4}+c}[ \\
& |C| \leq \frac{(m-s)\left(s-\frac{m^{2}}{n}+d\right)^{2}(n-1)(n+2)}{2\left(s-\frac{m^{2}}{n}\right)\left(-\left(s-\frac{m^{2}}{n}\right)^{2}+b\left(s-\frac{m^{2}}{n}\right)+c\right)}
\end{aligned}
$$




\section{The Endomorphisms $T_{k}$}

We introduce an endomorphism $T_{k}: S_{k} \rightarrow S_{k}$ which eigenvalues will play the role of the zeros of the zonal polynomials in the rank one case.

Proposition 4.1. Let

$$
\begin{aligned}
T_{k}: S_{k} & \rightarrow S_{k} \\
P & \mapsto \operatorname{pr}_{S_{k}}(\sigma P)
\end{aligned}
$$

where the orthogonal projection on $S_{k}$ is denoted by $\operatorname{pr}_{S_{k}}$ (note that, in general, $\sigma P$ does not belong to $S_{k}$ but rather to $S_{k+1}$ ).

The endomorphism $T_{k}$ is a symmetric endomorphism of $S_{k}$, and is an isomorphism.

Proof. We have, for all $P, Q \in S_{k},\left[T_{k}(P), Q\right]=[\sigma P, Q]=[P, \sigma Q]=$ $\left[P, T_{k}(Q)\right]$. Moreover, $[\sigma P, P]=\left[\sigma, P^{2}\right]>0$ unless $P=0$, because of the positivity of the measure on $\mathbb{R}\left[y_{1}, \ldots, y_{m}\right]^{S_{m}}$. Thus $T_{k}$ is injective.

Let $J_{k}$ be the matrix of this endomorphism in the basis $\left\{P_{\kappa},|\kappa| \leq k\right\}$. From the three-term relation (Theorem 2.4), $J_{k}$ is the block-tridiagonal matrix:

$$
J_{k}=\left(\begin{array}{cccccc}
B_{0} & A_{0} & & & & \\
C_{1} & B_{1} & A_{1} & & & \\
& C_{2} & B_{2} & A_{2} & & \\
& & C_{3} & \ddots & \ddots & \\
& & & \ddots & \ddots & A_{k-1} \\
& & & & C_{k} & B_{k}
\end{array}\right)
$$

It is worth noticing that the matrix $J_{k}$ itself is not symmetric, because the polynomials $P_{\kappa}$ are not of norm 1 . We shall later introduce and calculate the symmetric matrix $J^{\prime}{ }_{k}$ obtained in the normalized basis.

In the end, we shall need some very precise information on the coefficients of $J^{\prime}{ }_{k}$. For the moment, the only, but crucial, property that we will exploit is the fact that it is non-negative and irreducible.

Lemma 4.2. The eigenvalues of $T_{k}$ are real, and belong to $] 0, m[$. The maximal eigenvalue of $T_{k}$, denoted by $\lambda_{k}$, is of multiplicity 1 , and possesses an eigenvector with positive coordinates. Moreover, $\lambda_{k-1}<\lambda_{k}$.

Proof. The matrix $J_{k}$ is non-negative and irreducible in the sense of [22], because of Proposition 2.5 (note that the coefficients $A_{k}\left[\kappa, \kappa^{(i)}\right]$ are positive). Moreover, it is the matrix of a symmetric endomorphism, so its eigenvalues are real. From 22, Perron-Frobenius Theorem], it follows that the maximal eigenvalue has multiplicity equal to 1 , and that, if $v$ is an eigenvector, either 
$v$ or $-v$ has positive coordinates. Let us now prove that all its eigenvalues belong to $] 0, m[$.

For any $v \in S_{k}, v \neq 0$, we have $[\sigma v, v]=\int \sigma v^{2} d \mu(y)$, where $d \mu$ is a positive measure. We integrate on the domain $[0,1]^{m}$, on which $0 \leq \sigma \leq m$, hence $0<[\sigma v, v]<m[v, v]$. If $v$ is an eigenvector of $T_{k}$ associated with an eigenvalue $\lambda$, we have $[\sigma v, v]=[\lambda v, v]=\lambda[v, v]$, so we can conclude that $0<\lambda<m$.

Now let $v$ be an eigenvector of $T_{k-1}$ for $\lambda_{k-1}$, assumed to be of norm 1 . We have

$$
\sigma v=\lambda_{k-1} v+u
$$

with $u \in \Pi_{k}$. Obviously, since $\operatorname{deg}(\sigma v)=1+\operatorname{deg}(v), v$ must be of degree exactly $k-1$ (and $u \neq 0$ ).

Since

$$
\lambda_{k}=\max _{x \in S_{k} \backslash\{0\}} \frac{\left[T_{k}(x), x\right]}{[x, x]},
$$

we have $\left[T_{k}(v), v\right] \leq \lambda_{k}$. But $\left[T_{k}(v), v\right]=[\sigma v, v]=\lambda_{k-1}$. The equality $\lambda_{k-1}=\lambda_{k}$ would mean that $v$ is an eigenvector of $T_{k}$, which is not possible since it has degree $k-1$.

In the case $m=1$, the eigenvalues of $T_{k}$ are exactly the zeros of the polynomial $P_{k+1}$. In the general case, we prove in next lemma that common zeros of the polynomials $Q_{\kappa}$ give some of the eigenvalues. However, we do not know if such common zeros do exist, neither if all of the eigenvalues are obtained that way (and may be it is not so important):

Lemma 4.3. Let $\alpha \in[0,1]^{m}$ be a common zero of the polynomials

$$
Q_{\kappa}:=\sum_{|\mu|=k+1} A_{k}[\kappa, \mu] P_{\mu}
$$

for all $\kappa,|\kappa|=k$. Then, $v:=\sum_{|\nu| \leq k} d_{2 \nu} P_{\nu}(\alpha) P_{\nu}$ is an eigenvector of $T_{k}$ for the eigenvalue $\sigma(\alpha)$.

Proof. It is immediate from Christoffel-Darboux formula (Theorem 2.6(i)). If $Q_{\kappa}(\alpha)=0$ for all $\kappa,|\kappa|=k$, we have

$$
(\sigma(\alpha)-\sigma(y)) v=-\sum_{\substack{|\kappa|=k \\|\mu|=k+1}} d_{2 \kappa} A_{k}[\kappa, \mu] P_{\kappa}(\alpha) P_{\mu}(y) \in \Pi_{k+1}
$$

and, therefrom,

$$
\sigma(\alpha) v=T_{k}(v)
$$

We now show how to obtain a bound for the size of $\delta$-codes, as a function of $\delta$. Therefore, in order to cope with any possible $\delta$, we must perturb the endomorphism $T_{k}$ as explained next: 
Theorem 4.4. Let $\epsilon \in \mathbb{R}^{\pi_{k}}$, with $\epsilon_{\kappa} \geq 0$. Let $T_{k}^{\epsilon}$ be the endomorphism defined on $S_{k}$ by

$$
T_{k}^{\epsilon}(v)=T_{k}(v)-\epsilon * v_{k}
$$

where $\epsilon * v_{k}:=\sum_{|\kappa|=k} \epsilon_{\kappa} v_{\kappa} P_{\kappa}$.

(i) $T_{k}^{\epsilon}$ has a unique maximal eigenvalue $\lambda_{k}^{\epsilon}$, of multiplicity one, possessing an eigenvector $v^{\epsilon}$ with positive coefficients. Moreover, if $\epsilon \neq 0$,

$$
\lambda_{k-1}<\lambda_{k}^{\epsilon}<\lambda_{k}
$$

(ii) Let $\epsilon \neq 0$. Any $\delta$-code $C$ such that $s=m-\delta^{2}<\lambda_{k}^{\epsilon}$ satisfies

$$
|C| \leq \frac{\left(\sum_{|\kappa|=k} v_{\kappa}^{\epsilon}\left(\epsilon_{\kappa}+a_{\kappa}\right)\right)^{2}}{\left(m-\lambda_{k}^{\epsilon}\right)\left(\sum_{|\kappa|=k} d_{2 \kappa}^{-1} \epsilon_{\kappa} v_{\kappa}^{\epsilon 2}\right)}
$$

where $a_{\kappa}:=Q_{\kappa}(1, \ldots, 1)=\sum_{|\mu|=k+1} A_{k}[\kappa, \mu]$.

Proof. (i) The matrix $J_{k}^{\epsilon}$ of $T_{k}^{\epsilon}$ is equal to $J_{k}$, except the diagonal elements lying in $B_{k}$. Replacing $J_{k}^{\epsilon}$ by $J_{k}^{\epsilon}+M$ Id for some appropriate $M$, we obtain a non-negative matrix which is irreducible so its largest eigenvalue has multiplicity one and has an associated eigenvector with positive coordinates. It remains true for $J_{k}^{\epsilon}$. Since, when $\epsilon \neq 0, J_{k}^{\epsilon}<J_{k}$, we have $\lambda_{k}^{\epsilon}<\lambda_{k}$. The proof of the inequality $\lambda_{k-1}<\lambda_{k}^{\epsilon}$ is the same as the one of $\lambda_{k-1}<\lambda_{k}$.

(ii) We have $\sigma v^{\epsilon}=\lambda_{k}^{\epsilon} v^{\epsilon}+\epsilon * v_{k}^{\epsilon}+u$ where $u \in \Pi_{k+1}$. We need to compute $u$, and we set $u=\sum_{|\mu|=k+1} u_{\mu} P_{\mu}$. Let $\mu,|\mu|=k+1$, we have:

$$
\begin{aligned}
u_{\mu}\left[P_{\mu}, P_{\mu}\right] & =\left[u, P_{\mu}\right]=\left[\sigma v^{\epsilon}, P_{\mu}\right] \\
& =\sum_{|\kappa| \leq k} v_{\kappa}^{\epsilon}\left[\sigma P_{\kappa}, P_{\mu}\right] \\
& =\sum_{|\kappa|=k} v_{\kappa}^{\epsilon} A_{k}[\kappa, \mu]\left[P_{\mu}, P_{\mu}\right]
\end{aligned}
$$

and we obtain $u_{\mu}=\sum_{|\kappa|=k} v_{\kappa}^{\epsilon} A_{k}[\kappa, \mu]$. We have found

$$
u=\sum_{|\mu|=k+1}\left(\sum_{|\kappa|=k} v_{\kappa}^{\epsilon} A_{k}[\kappa, \mu]\right) P_{\mu}=\sum_{|\kappa|=k} v_{\kappa}^{\epsilon} Q_{\kappa},
$$

hence the "generalized Christoffel-Darboux formula":

$$
v^{\epsilon}=\sum_{s=0}^{k} v_{s}^{\epsilon} \mathbb{P}_{s}=\frac{\sum_{|\kappa|=k} v_{\kappa}^{\epsilon}\left(\epsilon_{\kappa} P_{\kappa}+Q_{\kappa}\right)}{\sigma-\lambda_{k}^{\epsilon}} .
$$


Now we proceed like in Proposition 2.8 Let the numerator of the right hand side be denoted by $N_{k+1}(y)$, and let

$$
F_{2 k+1}(y):=\frac{N_{k+1}(y)^{2}}{\sigma(y)-\lambda_{k}^{\epsilon}}=N_{k+1}(y) v^{\epsilon} .
$$

We have:

$$
\begin{aligned}
f_{0}=\left[F_{2 k+1}, 1\right] & =\left[N_{k+1}, v^{\epsilon}\right] \\
& =\left[\sum_{|\kappa|=k} v_{\kappa}^{\epsilon} \epsilon_{\kappa} P_{\kappa}, \sum_{|\kappa|=k} v_{\kappa}^{\epsilon} P_{\kappa}\right] \\
& =\sum_{|\kappa|=k} v_{\kappa}^{\epsilon} \epsilon_{\kappa} d_{2 \kappa}^{-1} .
\end{aligned}
$$

Since the coefficients of $\epsilon$ and of $v^{\epsilon}$ are non-negative numbers, and $f_{0} \neq 0$ when $\epsilon \neq 0$, it follows that $F_{2 k+1}$ satisfies the condition (i) of Proposition 2.3. Condition (ii) is clearly fulfilled if $s<\lambda_{k}^{\epsilon}$. We calculate

$$
F_{2 k+1}(1, \ldots, 1)=\frac{\left(\sum_{|\kappa|=k} v_{\kappa}^{\epsilon}\left(\epsilon_{\kappa}+a_{\kappa}\right)\right)^{2}}{m-\lambda_{k}^{\epsilon}} .
$$

hence the announced bound.

Let us show that we have indeed generalized the situation described in subsection 2.3 and Proposition 2.8. Let $x \in \mathbb{R}^{m}$ such that $P_{\kappa}(x)>0$, $Q_{\kappa}(x) \leq 0$ for all $|\kappa|=k$, and $P_{\kappa}(x) \geq 0$ for all $|\kappa| \leq k$. Let $\epsilon \in \mathbb{R}^{\pi_{k}}$ be defined by: $\epsilon_{\kappa}=-Q_{\kappa}(x) / P_{\kappa}(x)$. We can show that $\lambda_{k}^{\epsilon}=\sigma(x)$. Indeed, from (i) and (ii) of the proposition,

$$
v^{\epsilon}(y)=\frac{\sum_{|\kappa|=k} v_{\kappa}^{\epsilon}\left(-\left(Q_{\kappa}(x) / P_{\kappa}(x)\right) P_{\kappa}(y)+Q_{\kappa}(y)\right)}{\sigma(y)-\lambda_{k}^{\epsilon}} .
$$

When we let $y$ tend to $x$, the numerator tends to 0 . Since the coordinates of $v^{\epsilon}$ are positive and $P_{\kappa}(x) \geq 0$ for all $|\kappa| \leq k$, the left hand side cannot be equal to zero when $y=x\left(P_{0}=1\right)$. So the denominator also tends to zero, and $\lambda_{k}^{\epsilon}=\sigma(x)$. The Christoffel-Darboux formula (Theorem 2.6(i)) shows that $v^{\epsilon}=\sum_{|\kappa| \leq k} d_{2 \kappa} P_{\kappa}(x) P_{\kappa}$.

When $m=1, \pi_{k}=1$ and any $\epsilon \geq 0$ is of this form. When $m \geq 2$, it is not clear.. It is not even clear that at least one $x$ satisfying these inequalities exists.

Another natural question concerns the values that $\lambda_{k}^{\epsilon}$ takes. It is hoped of course that all values in the interval $\left.] \lambda_{k-1}, \lambda_{k}\right]$ are attained. We have defined a mapping from $\left[0,+\infty\left[{ }^{\pi_{k}}\right.\right.$ to $\left.] \lambda_{k-1}, \lambda_{k}\right]$, sending $\epsilon$ to $\lambda_{k}^{\epsilon}$, which is continuous, hence the image in an interval, containing $\lambda_{k}$, since clearly it is the image of $\epsilon=0$. Let us prove that $\lambda_{k}^{\epsilon}$ tends to $\lambda_{k-1}$ when $\epsilon$ tends to $+\infty$. To that end, we use the following inequality, valid for any non-negative 
matrix $\mathrm{J}$ with maximal eigenvalue $\lambda(22])$ :

$$
\text { For all } x, x_{i}>0, \lambda \leq \sup _{i} \frac{(x J)_{i}}{x_{i}} \text {. }
$$

This inequality remains true for the matrix $J_{k}^{\epsilon}$, although it is not nonnegative, because we can apply it to some $J_{k}^{\epsilon}+M \mathrm{Id}$, an argument that we have already called for. We choose for $x \in \mathbb{R}^{s_{k}}$ a vector, which first $s_{k-1}$ coefficients constitute a positive eigenvector of $J_{k-1}$ for the eigenvalue $\lambda_{k-1}$. Its last $\pi_{k}$ coordinates are denoted by $u=\left(u_{\kappa}\right)_{|\kappa|=k}$. We have:

$$
\left\{\begin{array}{l}
\text { If }|\nu| \leq k-2, \frac{\left(x J_{k}^{\epsilon}\right)_{\nu}}{x_{\nu}}=\lambda_{k-1} \\
\text { If }|\nu|=k-1, \frac{\left(x J_{k}^{\epsilon}\right)_{\nu}}{x_{\nu}}=\lambda_{k-1}+\frac{\sum_{|\kappa|=k} u_{\kappa} C_{k}[\kappa, \nu]}{x_{\nu}} \\
\text { If }|\kappa|=k, \frac{\left(x J_{k}^{\epsilon}\right)_{\kappa}}{x_{\kappa}}=\frac{\sum_{|\nu|=k-1} x_{\nu} A_{k-1}[\nu, \kappa]}{u_{\kappa}} \\
\quad+\left(B_{k}[\kappa, \kappa]-\epsilon_{\kappa}\right) .
\end{array}\right.
$$

The last equality relies on a result that is only proved in Section 5, Proposition [5.1(i), namely that $B_{k}\left[\kappa, \kappa^{\prime}\right]=0$ when $\kappa \neq \kappa^{\prime}$.

Let us now choose an arbitrary small $\alpha>0$; we can choose the coefficients $u_{\kappa}>0$ such that $\frac{\sum_{|\kappa|=k} u_{\kappa} C_{k}[\kappa, \nu]}{x_{\nu}} \leq \alpha$ for all $\nu$ with $|\nu|=k-1$. Then we can choose $\epsilon_{\kappa}>0$ such that $\frac{\sum_{|\nu|=k-1} x_{\nu} A_{k-1}[\nu, \kappa]}{u_{\kappa}}+\left(B_{k}[\kappa, \kappa]-\epsilon_{\kappa}\right)=0$. We are left with:

$$
\left\{\begin{array}{l}
\text { If }|\nu| \leq k-2, \frac{\left(x J_{k}^{\epsilon}\right)_{\nu}}{x_{\nu}}=\lambda_{k-1} \\
\text { If }|\nu|=k-1, \frac{\left(x J_{k}^{\epsilon}\right)_{\nu}}{x_{\nu}} \leq \lambda_{k-1}+\alpha \\
\text { If }|\kappa|=k, \frac{\left(x J_{k}^{\epsilon}\right)_{\kappa}}{x_{\kappa}}=0 .
\end{array}\right.
$$

Hence $\lambda_{k}^{\epsilon} \leq \lambda_{k-1}+\alpha$ for that choice of $\epsilon$.

Let us go back to the bound proved in Theorem 4.4. We can simplify further this bound, getting rid of the eigenvector. We obtain the following nicer, but weaker version:

Corollary 4.5. Let $C$ be a $\delta$-code such that $m-\delta^{2} \leq \lambda_{k-1}$. Then,

$$
|C| \leq \frac{4 \sum_{|\kappa|=k} d_{2 \kappa} a_{\kappa}}{m-\lambda_{k}}
$$

Proof. If $C$ satisfies $\delta^{2}>m-\lambda_{k-1}$, since $\lambda_{k-1}<\lambda_{k}^{\epsilon}$ for all non-negative $\epsilon$ (from Theorem 4.4 (i)), the bound of Theorem 4.4 (iv) applies to $C$. We get, using Cauchy-Schwartz inequality, and $\lambda_{k}^{\epsilon}<\lambda_{k}$ : 


$$
\begin{aligned}
|C| & \leq \frac{1}{m-\lambda_{k}^{\epsilon}} \frac{\left(\sum_{|\kappa|=k} v_{\kappa}^{\epsilon}\left(\epsilon_{\kappa}+a_{\kappa}\right)\right)^{2}}{\sum_{|\kappa|=k} d_{2 \kappa}^{-1} \epsilon_{\kappa} v_{\kappa}^{\epsilon}} \\
& \leq \frac{1}{m-\lambda_{k}} \sum_{|\kappa|=k} d_{2 \kappa} \frac{\left(\epsilon_{\kappa}+a_{\kappa}\right)^{2}}{\epsilon_{\kappa}} .
\end{aligned}
$$

The function $z \rightarrow \frac{(z+a)^{2}}{z}$ is minimized over $] 0,+\infty[$ when $z=a$. We obtain, with $\epsilon_{\kappa}=a_{\kappa}$, the announced bound.

\section{Asymptotic Behavior of the largest eigenvalue $\lambda_{k}$ of $T_{k}$}

In this section, we compute the limit taken by $\lambda_{k}$ when the quotient $n / k$ tends to some fixed value (Theorem [5.3). This result is needed to pass to the asymptotic in the inequality (9) for the size of a Grassmannian code.

We first need some very explicit formulas for the coefficients of the symmetric matrix $J^{\prime}{ }_{k}$ associated to the endomorphism $T_{k}$, in the orthonormal basis

$\left\{\sqrt{d_{2 \kappa}} P_{\kappa},|\kappa| \leq k\right\}$. From now on we change our usual convention: if not specified, $\kappa$ is a partition of degree $s$. The diagonal coefficients of ${J^{\prime}}_{k}$ are the same as the ones of $J_{k}$, while the other coefficients, denoted by $A^{\prime}{ }_{s}[\kappa, \mu]$, satisfy

$$
A^{\prime}{ }_{s}[\kappa, \mu]=A_{s}[\kappa, \mu] \sqrt{\frac{d_{2 \kappa}}{d_{2 \mu}}} .
$$

To start with, we gather some known results on the polynomials $C_{\kappa}$.

5.1. Review of some properties of the polynomials $C_{\kappa}$. The coefficients $\left[\begin{array}{l}\mu \\ \kappa\end{array}\right]$ and $\left(\begin{array}{l}\mu \\ \kappa\end{array}\right)$ are defined respectively by the following properties:

$$
\begin{aligned}
\sigma C_{\kappa} & =\sum_{|\mu|=s+1}\left[\begin{array}{l}
\mu \\
\kappa
\end{array}\right] C_{\mu} \\
\epsilon C_{\kappa} & =\sum_{|\nu|=s-1}\left(\begin{array}{l}
\kappa \\
\nu
\end{array}\right) C_{\nu}
\end{aligned}
$$

and have the following explicit expressions:

$$
\begin{aligned}
{\left[\begin{array}{c}
\kappa^{(i)} \\
\kappa
\end{array}\right] } & =\prod_{\substack{j=1 \\
j \neq i}}^{m} \frac{2 \kappa_{i}-2 \kappa_{j}+j-i+1}{2 \kappa_{i}-2 \kappa_{j}+j-i} \\
\left(\begin{array}{c}
\kappa^{(i)} \\
\kappa
\end{array}\right) & =\left(\kappa_{i}+1+\frac{m-i}{2}\right) \prod_{\substack{j=1 \\
j \neq i}}^{m} \frac{2 \kappa_{i}-2 \kappa_{j}+j-i+1}{2 \kappa_{j}+j-i+2}
\end{aligned}
$$


while any other values are equal to zero (see [17, Lemma 7.5.7], 17, 14, Th 14.1], [10]). The polynomials $C_{\kappa}$ are intimately related to the decomposition of $\mathrm{GL}(m, \mathbb{R})$-modules $(9$, Theorem 5.2.9]):

$$
\mathcal{R}(\mathrm{GL}(m, \mathbb{R}) / \mathrm{O}(m, \mathbb{R}))=\oplus_{\kappa} F_{m}^{2 \kappa} .
$$

For later use, we settle the notation: $\delta_{\kappa}:=\operatorname{dim}\left(F_{m}^{\kappa}\right)$ and we recall the formula $([8])$ :

$$
\delta_{\kappa}:=\operatorname{dim}\left(F_{m}^{\kappa}\right)=\prod_{1 \leq i<j \leq m} \frac{\kappa_{i}-\kappa_{j}+j-i}{j-i} .
$$

\subsection{Formulas for the coefficients of the matrix $J_{k}^{\prime}$.}

Proposition 5.1. The matrix $B_{s}$ has the following properties:

(i) $B_{s}\left[\kappa, \kappa^{\prime}\right]=0$ for all $\kappa \neq \kappa^{\prime}$.

(ii) If $m \leq n / 2$,

$$
\begin{aligned}
2 B_{s}[\kappa, \kappa]= & \sum_{i \in u(\kappa)}\left(\begin{array}{c}
\kappa^{(i)} \\
\kappa
\end{array}\right)\left[\begin{array}{c}
\kappa^{(i)} \\
\kappa
\end{array}\right] \frac{2 \kappa_{i}+m+1-i}{2 \kappa_{i}+n / 2+1-i} \\
& -\sum_{i \in d(\kappa)}\left(\begin{array}{c}
\kappa \\
\kappa_{(i)}
\end{array}\right)\left[\begin{array}{c}
\kappa \\
\kappa_{(i)}
\end{array}\right] \frac{2 \kappa_{i}+m-1-i}{2 \kappa_{i}+n / 2-1-i} .
\end{aligned}
$$

(iii) If $m=n / 2, B_{s}[\kappa, \kappa]=m / 2$.

Proof. We recall that the coefficients $\beta_{\kappa, \nu}$ are defined by:

$$
P_{\kappa}=\beta_{\kappa} C_{\kappa}+\sum_{\nu \mid \kappa>\nu} \beta_{\kappa, \nu} C_{\nu} .
$$

Inverting these relations, we obtain coefficients $\alpha_{\kappa, \nu}$ such that

$$
C_{\kappa}=\alpha_{\kappa} P_{\kappa}+\sum_{\nu \mid \kappa>\nu} \alpha_{\kappa, \nu} P_{\nu} .
$$

Taking into account the formula (10), we obtain:

$$
B_{s}\left[\kappa, \kappa^{\prime}\right]=\beta_{\kappa} \sum_{|\mu|=s+1}\left[\begin{array}{l}
\mu \\
\kappa
\end{array}\right] \alpha_{\mu, \kappa^{\prime}}+\sum_{|\nu|=s-1} \beta_{\kappa, \nu}\left[\begin{array}{c}
\kappa^{\prime} \\
\nu
\end{array}\right] \alpha_{\kappa^{\prime}} .
$$

We use the following obvious relations: $\alpha_{\kappa} \beta_{\kappa}=1$ and $\alpha_{\kappa} \beta_{\kappa, \kappa^{\prime}}+\beta_{\kappa^{\prime}} \alpha_{\kappa, \kappa^{\prime}}=$ 0 to rewrite

$$
\begin{aligned}
B_{s}\left[\kappa, \kappa^{\prime}\right]=\frac{\beta_{\kappa}}{\beta_{\kappa^{\prime}}}\left(-\sum_{|\mu|=s+1}\left[\begin{array}{l}
\mu \\
\kappa
\end{array}\right] \frac{\beta_{\mu, \kappa^{\prime}}}{\beta_{\mu}}\right. & \left.+\sum_{|\nu|=s-1}\left[\begin{array}{c}
\kappa^{\prime} \\
\nu
\end{array}\right] \alpha_{\kappa^{\prime}} \frac{\beta_{\kappa, \nu}}{\beta_{\kappa}}\right) .
\end{aligned}
$$


Let us assume first that $\kappa \neq \kappa^{\prime}$. Since $\left[\begin{array}{l}\mu \\ \kappa\end{array}\right]$ is non zero only if $\mu=\kappa^{(i)}$ for some index $i$, and also $\beta_{\mu, \kappa^{\prime}}$ is non zero only if $\mu=\kappa^{\prime(j)}$ for some index $j$, at most one term in the first summation may be non zero, and the same argument holds for the second summation. We only have to consider the case when $\kappa^{\prime}$ satisfies: for some indexes $i \neq j, \kappa_{i}^{\prime}=\kappa_{i}+1$ and $\kappa_{j}^{\prime}=\kappa_{j}-1$. The remaining terms in the expression of $B_{s}\left[\kappa, \kappa^{\prime}\right]$ correspond to $\mu=\kappa^{(i)}=\kappa^{(j)}$ and $\nu=\kappa_{(j)}=\kappa_{(i)}^{\prime}$.

Moreover, the coefficients $\beta_{\mu, \kappa}$ are calculated in [14, and in particular we have:

$$
\frac{\beta_{\kappa, \kappa_{(j)}}}{\beta_{\kappa}}=-\frac{1}{2}\left(\begin{array}{c}
\kappa \\
\kappa_{(j)}
\end{array}\right) \frac{2 \kappa_{j}+m-1-j}{2 \kappa_{j}+n / 2-1-j} .
$$

Replacing in (15) we have

$$
\begin{aligned}
B_{s}\left[\kappa, \kappa^{\prime}\right]=\frac{\beta_{\kappa}}{\beta_{\kappa^{\prime}}} & \frac{2 \kappa_{j}+m-1-j}{2\left(2 \kappa_{j}+n / 2-1-j\right)} . \\
& \left(\left[\begin{array}{c}
\kappa^{(i)} \\
\kappa
\end{array}\right]\left(\begin{array}{c}
\kappa^{(i)} \\
\kappa^{\prime}
\end{array}\right)-\left(\begin{array}{c}
\kappa \\
\kappa_{(j)}
\end{array}\right)\left[\begin{array}{c}
\kappa^{\prime} \\
\kappa_{(j)}
\end{array}\right]\right) .
\end{aligned}
$$

Combining (10) and (11) in the obvious relation:

$$
(\epsilon \sigma-\sigma \epsilon) C_{\kappa}=m C_{\kappa}
$$

leads to:

$$
\sum_{|\mu|=s+1}\left[\begin{array}{l}
\mu \\
\kappa
\end{array}\right]\left(\begin{array}{c}
\mu \\
\kappa^{\prime}
\end{array}\right)-\sum_{|\nu|=s-1}\left(\begin{array}{l}
\kappa \\
\nu
\end{array}\right)\left[\begin{array}{l}
\kappa^{\prime} \\
\nu
\end{array}\right]=\left\{\begin{array}{l}
0 \text { if } \kappa \neq \kappa^{\prime} \\
m \text { if } \kappa=\kappa^{\prime}
\end{array}\right.
$$

From (18) we can conclude that $B_{s}\left[\kappa, \kappa^{\prime}\right]=0$ when $\kappa \neq \kappa^{\prime}$.

When $\kappa=\kappa^{\prime}$, replacing (17) in (15) leads to the formula (ii). If moreover $n=m / 2$, taking account of (18) we obtain $B_{s}[\kappa, \kappa]=m / 2$.

We now give explicit formulas for the coefficients of $J^{\prime}{ }_{k}$ :

Proposition 5.2. With the following notations:

$$
\begin{gathered}
q_{i}:=2 \kappa_{i}-i+m \\
N:=n-2 m \\
D(x)=\frac{x^{2}}{x^{2}-1} \quad \text { and } \\
\left\{\begin{array}{l}
C(x)=\frac{(x+1)(x+N)}{(2 x+N)(2 x+N+2)} \quad x \neq 0 \\
C(0)=\frac{1}{N+2}
\end{array}\right.
\end{gathered}
$$


we have the expressions:

$$
\begin{aligned}
& B_{s}[\kappa, \kappa]= \\
& \frac{m}{2}-\frac{N}{4} \sum_{i \in u(\kappa)}\left(\prod_{\substack{j=1 \\
j \neq i}}^{m} D\left(q_{i}-q_{j}+1\right)\right) \frac{q_{i}+2}{2 q_{i}+N+2} \\
&+\frac{N}{4} \sum_{i \in d(\kappa)}\left(\prod_{\substack{j=1 \\
j \neq i}}^{m} D\left(q_{i}-q_{j}-1\right)\right) \frac{q_{i}}{2 q_{i}+N-2} . \\
& A^{\prime}{ }_{s}\left[\kappa, \kappa^{(i)}\right]=\left(\left(\prod_{j \neq i} D\left(q_{i}-q_{j}+1\right) D\left(q_{i}+q_{j}+N+1\right)\right) .\right. \\
&\left.C\left(q_{i}\right) C\left(q_{i}+1\right)\right)^{1 / 2} .
\end{aligned}
$$

Proof. For the calculation of $B_{s}$, we replace in Proposition 5.1 (ii) the formulas (12) and (13), and take account of Proposition 5.1 (iii).

In order to calculate $A^{\prime}{ }_{s}\left[\kappa, \kappa^{(i)}\right]$, we have already seen that:

$$
A_{s}\left[\kappa, \kappa^{(i)}\right]=\left[\begin{array}{c}
\kappa^{(i)} \\
\kappa
\end{array}\right]\left(\frac{\beta_{\kappa^{(i)}}}{\beta_{\kappa}}\right)^{-1} .
$$

We need a formula for $\left(\frac{\beta_{\kappa}(i)}{\beta_{\kappa}}\right)^{-1}$. Expressions for the leading coefficients of the polynomials $C_{\kappa}$ and $P_{\kappa}$ can be found in [12] and [23]. Putting them together we find:

$$
\frac{\beta_{\kappa^{(i)}}}{\beta_{\kappa}}=\prod_{\substack{j=1 \\ j \neq i}}^{m}\left(\frac{q_{i}+q_{j}+N}{q_{i}+q_{j}+N+1}\right) \frac{\left(2 q_{i}+N\right)\left(2 q_{i}+N+2\right)}{\left(q_{i}+N\right)\left(q_{i}+N+1\right)}
$$

where the last fraction must be understood as $(N+2) /(N+1)$ when $q_{i}=0$. Joined with (10), we obtain

$$
\begin{aligned}
A_{s}\left[\kappa, \kappa^{(i)}\right]=\prod_{\substack{j=1 \\
j \neq i}}^{m}\left(\frac{q_{i}-q_{j}+1}{q_{i}-q_{j}}\right)\left(\frac{q_{i}+q_{j}+N+1}{q_{i}+q_{j}+N}\right) . \\
\frac{\left(q_{i}+N\right)\left(q_{i}+N+1\right)}{\left(2 q_{i}+N\right)\left(2 q_{i}+N+2\right)} .
\end{aligned}
$$

Next we use ([8]):

$$
\begin{array}{r}
\frac{d_{2 \kappa^{(i)}}}{d_{2 \kappa}}=\prod_{\substack{j=1 \\
j \neq i}}^{m}\left(\frac{q_{i}-q_{j}+2}{q_{i}-q_{j}}\right)\left(\frac{q_{i}+q_{j}+N+2}{q_{i}+q_{j}+N}\right) . \\
\frac{\left(2 q_{i}+N+4\right)\left(q_{i}+N\right)\left(q_{i}+N+1\right)}{\left(2 q_{i}+N\right)\left(q_{i}+1\right)\left(q_{i}+2\right)}
\end{array}
$$


where the last fraction must be understood as $(N+4)(N+2) / 2$ when $q_{i}=0$, and we obtain the announced formula for $A^{\prime}\left[\kappa, \kappa^{(i)}\right]$.

5.3. The limit of $\lambda_{k}$. Now $n$ varies with $k$ so we rather denote by $T_{k}^{(n)}$ the endomorphism defined previously and $\lambda_{k}^{(n)}$ its largest eigenvalue.

Theorem 5.3. If $n / 2 k \rightarrow \ell$, while $n \rightarrow+\infty$ and $k \rightarrow+\infty$,

$$
\lim \lambda_{k}^{(n)}=4 \frac{\ell+1 / m}{(\ell+2 / m)^{2}} .
$$

Proof. We give careful proofs in the cases $m=1$ and $m=2$, and will be more sketchy in the general case. As it was noticed previously, when $m=1$ the eigenvalues are the zeros of the Jacobi polynomials; their asymptotic is calculated in [7, exploiting the differential equation for the Jacobi polynomials and Sturm's method. Another approach, using chain sequences, is used in 24]. However, none of these methods seem to generalize easily to the several variable case. Our argument will only use the fact that the matrix $J_{k}^{\prime(n)}$ is non-negative. More precisely, we use the following:

Lemma 5.4. 22 Let $J$ be a non-negative symmetric matrix of size $N$, with largest eigenvalue $\lambda$.

(i) For all $x \in \mathbb{R}^{N}$ with $x_{i}>0, \lambda \leq \max _{i} \frac{(x J)_{i}}{x_{i}}$.

(ii) For all $x \in \mathbb{R}^{N} x \neq 0, \lambda \geq \frac{(x J) \cdot x}{x \cdot x}$.

The case $m=1$. We recover from Proposition 5.2 the formulas:

$$
\begin{aligned}
2 b_{s} & =1-\frac{(n-2)(n-4)}{(4 s+n)(4 s+n-4)} \\
a_{s}^{\prime} & =\left(\frac{(2 s+1)(2 s+2)(2 s+n-2)(2 s+n-1)}{(4 s+n-2)(4 s+n)^{2}(4 s+n+2)}\right)^{1 / 2}
\end{aligned}
$$

From these expressions we see that both sequences are increasing with $s$. Moreover, we see easily that if $s \sim k, b_{s} \sim 2 \frac{\ell+1}{(\ell+2)^{2}}$, and $a_{s}^{\prime} \sim \frac{\ell+1}{(\ell+2)^{2}}$. Applying Lemma 5.4 (i) with $x_{s}=1$ for all $s$ leads to:

$$
\lambda_{k}^{(n)} \leq a^{\prime}{ }_{k-1}+b_{k}+a_{k}^{\prime}
$$

and the right hand side tends to $4(\ell+1) /(\ell+2)^{2}$ when $n / 2 k$ tends to $\ell$.

We lower bound $\lambda_{k}^{(n)}$ using Lemma 5.4 (ii) and a choice of $x$ proposed in 24]: let $x$ be defined by:

$$
\left\{\begin{array}{ccc}
x_{s}=0 & 1 \leq s \leq t:=k-\lfloor\sqrt{k}\rfloor+1 \\
x_{s}=1 & t+1 \leq s \leq k+1
\end{array}\right.
$$

so that $x_{s}=1$ on the $\lfloor\sqrt{k}\rfloor$ last coordinates. Then, 


$$
\begin{aligned}
\frac{\left(x{J_{k}^{\prime}}_{k}^{(n)}\right) \cdot x}{x \cdot x} & \geq \frac{\sum_{s=t+1}^{k-1}\left(a_{s-1}^{\prime}+b_{s}+a_{s}^{\prime}\right)}{k-t+1} \\
& \geq\left(\frac{k-t-1}{k-t+1}\right)\left(a_{t}^{\prime}+b_{t+1}+a_{t+1}^{\prime}\right)
\end{aligned}
$$

Again, the right hand side tends to $4(\ell+1) /(\ell+2)^{2}$, hence the result.

The case $m=2$. From Proposition 5.2 we have, setting $s:=\kappa_{1}+\kappa_{2}$ and $v:=\kappa_{1}-\kappa_{2}$ :

$$
\begin{gathered}
B_{s}[\kappa, \kappa]=1+\frac{(n-6)(n-4)}{8(4 s+2 n-6)}\left(\frac{(4 s+2 n-4)^{2}}{\left(4 \kappa_{1}+n\right)\left(4 \kappa_{2}+n-2\right)}\right. \\
\left.-\frac{(4 s+2 n-8)^{2}}{\left(4 \kappa_{1}+n-4\right)\left(4 \kappa_{2}+n-6\right)}\right) \\
A^{\prime}{ }_{s}\left[\kappa, \kappa^{(1)}\right]=\left(\frac{(2 v+2)^{2}}{(2 v+2)^{2}-1}\right)^{1 / 2}\left(\frac{(2 s+n-2)^{2}}{(2 s+n-2)^{2}-1}\right)^{1 / 2} . \\
\left(\frac{\left(2 \kappa_{1}+2\right)\left(2 \kappa_{1}+3\right)\left(2 \kappa_{1}+n-3\right)\left(2 \kappa_{1}+n-2\right)}{\left(4 \kappa_{1}+n-2\right)\left(4 \kappa_{1}+n\right)^{2}\left(4 \kappa_{1}+n+2\right)}\right)^{1 / 2} \\
A^{\prime}{ }_{s}\left[\kappa, \kappa^{(2)}\right]=\left(\frac{(2 v)^{2}}{(2 v)^{2}-1}\right)^{1 / 2}\left(\frac{(2 s+n-2)^{2}}{(2 s+n-2)^{2}-1}\right)^{1 / 2} \cdot \\
\left(\frac{\left(2 \kappa_{2}+1\right)\left(2 \kappa_{2}+2\right)\left(2 \kappa_{2}+n-4\right)\left(2 \kappa_{2}+n-3\right)}{\left(4 \kappa_{2}+n-4\right)\left(4 \kappa_{2}+n-2\right)^{2}\left(4 \kappa_{2}+n\right)}\right)^{1 / 2}
\end{gathered}
$$

One can verify that these coefficients are increasing with $s$ when $v$ stays constant. This is easy to see for $B_{s}$, not so obvious for the two others because the second term is decreasing while the last big quotient is increasing.

In order to obtain a lower bound for $\lambda_{k}^{(n)}$ from Lemma 5.4 (ii), we choose $x=\left(x_{\kappa}\right)$ with: $x_{\kappa}=0,1$. We fix a number $V<k-\sqrt{k}$. Let $\mathcal{K}_{V, s}$ be the set of the $V$ partitions of degree $s$ with smallest $v=\kappa_{1}-\kappa_{2}$. Hence $\mathcal{K}_{V, s}=\{\kappa \mid$ $\left.|\kappa|=s, \kappa_{2} \geq\left\lfloor\frac{s}{2}\right\rfloor-V+1\right\}$. We set $x_{\kappa}=1$ iff $\operatorname{deg}(\kappa) \geq t:=k-\lfloor\sqrt{k}\rfloor+1$, and $\kappa \in \mathcal{K}_{V,|\kappa|}$. We need to avoid in $\mathcal{K}_{V, s}$ some partitions, namely the ones with $v=0$ and the ones with $v$ maximal (for those partitions, some terms are either missing or are equal to zero in $\left.\left(x{J^{\prime}}_{k}^{(n)}\right)_{\kappa}\right)$. Let this new set be denoted by $\mathcal{K}_{V, s}^{\prime}$. We have, when $x_{\kappa}=1, \kappa \in \mathcal{K}_{V, s}^{\prime},|\kappa| \neq t, k$,

$$
\begin{aligned}
\left(x{J^{\prime}}_{k}^{(n)}\right)_{\kappa}=B_{s}[\kappa, \kappa] & +\sum_{i=1}^{2} A^{\prime}{ }_{s}\left[\kappa, \kappa^{(i)}\right] \\
& +\sum_{i=1}^{2} A^{\prime}{ }_{s-1}\left[\kappa_{(i)}, \kappa\right]
\end{aligned}
$$


In the expressions of $A^{\prime}{ }_{s}\left[\kappa, \kappa^{(i)}\right]$ we can minor the first term by $1(v \neq 0)$, then minor each term by its minimal value in the sequence $v=$ cte to which it belongs. As was mentioned before, this minimal value is obtained when the degree is minimal, i.e. when $s=t$ or $s=t+1$. We do the same for $A^{\prime}{ }_{s-1}\left[\kappa_{(i)}, \kappa\right]$ and for $B_{s}[\kappa, \kappa]$. Then we must consider the behavior when $s$ is constant of $B_{s}[\kappa, \kappa]$, of:

$$
\begin{aligned}
A_{s}^{1}[\kappa]:= & \frac{(2 s+n-2)}{\left((2 s+n-2)^{2}-1\right)^{1 / 2}} . \\
& \left(\left(C\left(q_{1}\right) C\left(q_{1}+1\right)\right)^{1 / 2}+\left(C\left(q_{2}\right) C\left(q_{2}+1\right)\right)^{1 / 2}\right)
\end{aligned}
$$

and of the analogous expression $A^{2}{ }_{s-1}[\kappa]$ corresponding to the last term. These expressions are increasing with $\kappa_{2}$. Let $B_{s, V}{ }^{\min }, A^{i}{ }_{s, V}{ }^{\text {min }}$ be their minimal values in $\mathcal{K}_{V, s}^{\prime}$. For simplicity, we assume that

$$
\min \left(B_{t, V}^{\min }, B_{t+1, V}^{\min }\right)=B_{t, V}^{\min }
$$

and the same for $A^{1}, A^{2}$.

We obtain:

$$
\begin{aligned}
& \frac{\left(x{J^{\prime}}_{k}^{(n)}\right) \cdot x}{x \cdot x} \geq \\
& \frac{(k-t-1)(V-2)}{(k-t+1) V}\left(B_{t, V}{ }^{\text {min }}+A^{1}{ }_{t, V}{ }^{\text {min }}+A^{2}{ }_{t, V}{ }^{\text {min }}\right) .
\end{aligned}
$$

Now we let $n / 2 k$ tend to $\ell$. Since $B_{t, V}$ min is obtained at a partition essentially equal to $[t / 2-V / 2, t / 2+V / 2$, and since $t \sim k$, we see that $B_{t, V}{ }^{\text {min }}$ tends to $2(\ell+1 / 2) /(\ell+1)^{2}$. For the same reason, $A^{1}{ }_{t, V}$ min and $A^{2}{ }_{t, V}{ }^{\text {min }}$ tend to $(\ell+1 / 2) /(\ell+1)^{2}$ (the parameter $V$ is still fixed at this stage). So we obtain

$$
\liminf \lambda_{k}^{(n)} \geq\left(1-\frac{2}{V}\right) \cdot 4 \frac{\ell+1 / 2}{(\ell+1)^{2}}
$$

Now we let $V$ tend to $+\infty$ to obtain the appropriate lower bound.

The second and last step obtains an upper bound for $\lambda_{k}^{(n)}$ from Lemma 5.4 (i) with an appropriate choice of $x$. The choice $x_{\kappa}=1$ for all $\kappa$ is not good enough here because $D(2 v+2)^{1 / 2}+D(2 v)^{1 / 2} \neq 2$. We need some $x_{\kappa}$ that modify properly these factors. We choose $x_{\kappa}:=(2 v+1)^{1 / 2}$ where $v=\kappa_{1}-\kappa_{2}$. We have 


$$
\begin{aligned}
\frac{\left(x{J^{\prime}}_{k}^{(n)}\right)_{\kappa}}{x_{\kappa}}=B_{s}[\kappa, \kappa] & +\sum_{i=1}^{2} A^{\prime}{ }_{s}\left[\kappa, \kappa^{(i)}\right] \frac{x_{\kappa^{(i)}}}{x_{\kappa}} \\
& +\sum_{i=1}^{2} A^{\prime}{ }_{s-1}\left[\kappa_{(i)}, \kappa\right] \frac{x_{\kappa_{(i)}}}{x_{\kappa}}
\end{aligned}
$$

Let:

$$
\begin{gathered}
A^{\sharp}\left[\kappa, \kappa^{(1)}\right]=\left(\frac{2 v+2}{2 v+1}\right)\left(\frac{(2 s+n-2)^{2}}{(2 s+n-2)^{2}-1}\right)^{1 / 2} \cdot \\
\left(\frac{\left(2 \kappa_{1}+2\right)\left(2 \kappa_{1}+3\right)\left(2 \kappa_{1}+n-3\right)\left(2 \kappa_{1}+n-2\right)}{\left(4 \kappa_{1}+n-2\right)\left(4 \kappa_{1}+n\right)^{2}\left(4 \kappa_{1}+n+2\right)}\right)^{1 / 2} \\
A^{\sharp}\left[\kappa, \kappa^{(2)}\right]=\left(\frac{2 v}{2 v+1}\right)\left(\frac{(2 s+n-2)^{2}}{(2 s+n-2)^{2}-1}\right)^{1 / 2} \cdot \\
\left(\frac{\left(2 \kappa_{2}+1\right)\left(2 \kappa_{2}+2\right)\left(2 \kappa_{2}+n-4\right)\left(2 \kappa_{2}+n-3\right)}{\left(4 \kappa_{2}+n-4\right)\left(4 \kappa_{2}+n-2\right)^{2}\left(4 \kappa_{2}+n\right)}\right)^{1 / 2} \\
A^{b}\left[\kappa_{(1)}, \kappa\right]=\left(\frac{2 v}{2 v+1}\right)\left(\frac{(2 s+n-4)^{2}}{(2 s+n-4)^{2}-1}\right)^{1 / 2} \cdot \\
\left(\frac{\left(2 \kappa_{1}\right)\left(2 \kappa_{1}+1\right)\left(2 \kappa_{1}+n-5\right)\left(2 \kappa_{1}+n-4\right)}{\left(4 \kappa_{1}+n-6\right)\left(4 \kappa_{1}+n-4\right)^{2}\left(4 \kappa_{1}+n-2\right)}\right)^{1 / 2} \\
A^{b}\left[\kappa_{(2)}, \kappa\right]=\left(\frac{2 v+2}{2 v+1}\right)\left(\frac{(2 s+n-4)^{2}}{(2 s+n-4)^{2}-1}\right)^{1 / 2} \cdot \\
\left(\frac{\left(2 \kappa_{2}-1\right)\left(2 \kappa_{2}\right)\left(2 \kappa_{2}+n-6\right)\left(2 \kappa_{2}+n-5\right)}{\left(4 \kappa_{2}+n-8\right)\left(4 \kappa_{2}+n-6\right)^{2}\left(4 \kappa_{2}+n-4\right)}\right)^{1 / 2}
\end{gathered}
$$

Since

and

$$
D(2 v+2)^{1 / 2} \frac{(2 v+3)^{1 / 2}}{(2 v+1)^{1 / 2}}=\frac{2 v+2}{2 v+1},
$$

we have:

$$
D(2 v)^{1 / 2} \frac{(2 v-1)^{1 / 2}}{(2 v+1)^{1 / 2}}=\frac{2 v}{2 v+1},
$$

$$
\begin{aligned}
\frac{\left(x{J_{k}^{\prime}}_{k}^{(n)}\right)_{\kappa}}{x_{\kappa}}=B_{s}[\kappa, \kappa] & +\sum_{i=1}^{2} A^{\sharp}\left[\kappa, \kappa^{(i)}\right] \\
& +\sum_{i=1}^{2} A^{b}\left[\kappa_{(i)}, \kappa\right] .
\end{aligned}
$$

This expression is increasing with $s$ when $v$ is fixed. When $s$ is fixed, $B_{s}[\kappa, \kappa], \sum_{i=1}^{2} A^{\sharp}\left[\kappa, \kappa^{(i)}\right]$ and $\sum_{i=1}^{2} A^{b}\left[\kappa_{(i)}, \kappa\right]$ are maximal at $\kappa=[s / 2, s / 2]$ 
(we extend the functions to partitions with real parts here). We obtain, with $\rho_{k}=[k / 2, k / 2]$,

$$
\begin{aligned}
\max _{\kappa} \frac{\left(x{J_{k}^{\prime}}_{k}^{(n)}\right)_{\kappa}}{x_{\kappa}} & \leq \\
B_{s}\left[\rho_{k}, \rho_{k}\right] & +\sum_{i=1}^{2} A^{\sharp}\left[\rho_{k}, \rho_{k}{ }^{(i)}\right]+\sum_{i=1}^{2} A^{b}\left[\rho_{k(i)}, \rho_{k}\right] .
\end{aligned}
$$

The computation of these values shows that the right hand side tends to $4(\ell+1 / 2) /(\ell+1)^{2}$ when $n / 2 k \rightarrow \ell$.

The general case $\mathbf{m}>\mathbf{2}$ works the same. For the lower bound, we use $\mathcal{K}_{V, s}:=\left\{\kappa|| \kappa \mid=s, \kappa_{m} \geq\left\lfloor\frac{s}{m}\right\rfloor-V+1\right\}$. The cardinality of $\mathcal{K}_{V, s}$ only depends on $s \bmod m$. We should avoid some partitions in $\mathcal{K}_{V, s}$, namely the ones with some parts equal and the ones with $\kappa_{m}=\left\lfloor\frac{s}{m}\right\rfloor-V+1$. Their number is negligible compared to the cardinality of $\mathcal{K}_{V, s}$. Then, we proceed in the same way as for $m=2$.

The upper bound is obtained with $x_{\kappa}=\left(\delta_{2 \kappa}\right)^{1 / 2}$. We have

$$
\prod_{\substack{j=1 \\ j \neq i}}^{m} D\left(q_{i}-q_{j}+1\right)^{1 / 2}\left(\frac{\delta_{2 \kappa^{(i)}}}{\delta_{2 \kappa}}\right)^{1 / 2}=\prod_{\substack{j=1 \\ j \neq i}}^{m} \frac{q_{i}-q_{j}+1}{q_{i}-q_{j}} .
$$

hence

$$
\begin{aligned}
\frac{\left(x{J_{k}^{\prime}}_{k}^{(n)}\right)_{\kappa}}{x_{\kappa}}=B_{s}[\kappa, \kappa] & +\sum_{i=1}^{m} A^{\sharp}\left[\kappa, \kappa^{(i)}\right] \\
& +\sum_{i=1}^{m} A^{b}\left[\kappa_{(i)}, \kappa\right],
\end{aligned}
$$

where

$$
\begin{aligned}
A^{\sharp}\left[\kappa, \kappa^{(i)}\right] & =\prod_{\substack{j=1 \\
j \neq i}}^{m} \frac{q_{i}-q_{j}+1}{q_{i}-q_{j}} . \\
& \left(\left(\prod_{\substack{j=1 \\
j \neq i}}^{m} D\left(q_{i}+q_{j}+N+1\right)\right) C\left(q_{i}\right) C\left(q_{i}+1\right)\right)^{1 / 2}
\end{aligned}
$$


and similarly

$$
\begin{aligned}
A^{\mathrm{b}}\left[\kappa_{(i)}, \kappa\right] & =\prod_{\substack{j=1 \\
j \neq i}}^{m} \frac{q_{i}-q_{j}-1}{q_{i}-q_{j}} . \\
& \left(\left(\prod_{\substack{j=1 \\
j \neq i}}^{m} D\left(q_{i}+q_{j}+N-1\right)\right) C\left(q_{i}-2\right) C\left(q_{i}-1\right)\right)^{1 / 2} .
\end{aligned}
$$

We have the nice identity:

$$
\sum_{i=1}^{m} \prod_{\substack{j=1 \\ j \neq i}}^{m} \frac{q_{i}-q_{j}+1}{q_{i}-q_{j}}=m
$$

We do not have a reference for this last identity, so we give an argument here: from (14),

$$
\frac{\operatorname{dim} F_{m}^{\kappa^{(i)}}}{\operatorname{dim} F_{m}^{\kappa}}=\prod_{\substack{j=1 \\ j \neq i}}^{m} \frac{\kappa_{i}-\kappa_{j}+j-i+1}{\kappa_{i}-\kappa_{j}+j-i}
$$

We obtain the demanded identity as the equality of the dimensions in the following decomposition of $\mathrm{GL}(m, \mathbb{R})$-modules (Pieri's rule, 9$]$ ):

$$
F_{m}^{(1)} \otimes F_{m}^{\kappa}=\oplus_{i=1}^{m} F_{m}^{\kappa^{(i)}} .
$$

It turns out that the coefficients $B[\kappa, \kappa], A^{\sharp}\left[\kappa, \kappa^{(i)}\right]$ and $A^{b}\left[\kappa_{(i)}, \kappa\right]$ are increasing when $\kappa$ runs over a sequence of the type $(\nu+s[1,1, \ldots, 1])_{s \geq 0}$ (when $N$ is big enough), and that, on the space of partitions (with real parts) $\kappa$ of fixed degree $k$, the maximum of the expressions $B[\kappa, \kappa], \sum_{i=1}^{m} A^{\sharp}\left[\kappa, \kappa^{(i)}\right]$ and $\sum_{i=1}^{m} A^{b}\left[\kappa_{(i)}, \kappa\right]$ is attained at $\kappa=\rho_{k}=[k / m, k / m, \ldots, k / m]$.

Moreover, it is easy to see that, when $n / 2 k \rightarrow \ell$,

$$
\begin{aligned}
\lim B_{k}\left[\rho_{k}, \rho_{k}\right] & =2 \frac{\ell+1 / m}{(\ell+2 / m)^{2}} \\
\lim \left(\sum_{i=1}^{m} A^{\sharp}\left[\rho_{k}, \rho_{k}^{(i)}\right]\right) & =\lim \left(\sum_{i=1}^{m} A^{b}\left[\left(\rho_{k}\right)_{(i)}, \rho_{k}\right]\right) \\
& =\frac{\ell+1 / m}{(\ell+2 / m)^{2}} .
\end{aligned}
$$

Remark 5.5. One obvious consequence of Theorem 5.3 is that, for fixed $n$, the eigenvalue $\lambda_{k}^{(n)}$ runs over the whole interval $] 0, m[$. Hence the bounds proved in section 4 for the size of grassmannian codes potentially cover all possible minimal distance. 


\section{An ASYmptotic Bound for the Size of Grassmannian CODES}

We are now ready to take the limit when $n$ tends to $+\infty$ in the inequality (9), and prove Theorem 1.1

We are left with the estimate of $\log \left(\sum_{|\kappa|=k} d_{2 \kappa} a_{\kappa}\right) / n$.

Lemma 6.1. Let $\delta_{\kappa}:=\operatorname{dim}\left(F_{n}^{\kappa}\right)$. If $n / 2 k \rightarrow \rho^{-1} \in \mathbb{R}$ while $n$ and $k$ tend to $+\infty$

$$
\begin{aligned}
\limsup & \frac{1}{n} \log \left(\sum_{\substack{|\kappa|=2 k \\
\ell(\kappa) \leq m}} \delta_{\kappa}\right) \leq \\
& m((1+\rho) \log (1+\rho)-\rho \log (\rho)) .
\end{aligned}
$$

Proof. In the case $m=1, \delta_{2 k}=\operatorname{dim} S_{2 k}=\left(\begin{array}{c}n+2 k-1 \\ 2 k\end{array}\right)$ and it is a classical result. The general case is probably well-known but since we lack a reference, we give a proof here. Let $\kappa$ be a partition of length at most $m$ and of degree $2 k$, that we extend to a partition with $n$ parts with an appropriate number of zeros. From (14),

$$
\operatorname{dim}\left(F_{n}^{\kappa}\right)=\prod_{1 \leq i<j \leq n} \frac{\kappa_{i}-\kappa_{j}+j-i}{j-i} .
$$

Since $\kappa_{j}=0$ when $j>m$, we have

$$
\operatorname{dim}\left(F_{n}^{\kappa}\right)=\prod_{1 \leq i<j \leq m} \frac{\kappa_{i}-\kappa_{j}+j-i}{j-i} \prod_{1 \leq i \leq m} \prod_{j>m} \frac{\kappa_{i}+j-i}{j-i}
$$

We upper bound:

$$
\prod_{j>m} \frac{\kappa_{i}+j-i}{j-i} \leq\left(\begin{array}{c}
n+\kappa_{i}-1 \\
\kappa_{i}
\end{array}\right)
$$

and

to obtain

$$
\prod_{1 \leq i<j \leq m} \frac{\kappa_{i}-\kappa_{j}+j-i}{j-i} \leq(2 k+1)^{m^{2}}
$$

$$
\begin{aligned}
\sum_{\substack{|\kappa|=2 k \\
\ell(\kappa) \leq m}} \delta_{\kappa} & \leq(2 k+1)^{m^{2}} \sum_{\substack{|\kappa|=2 k \\
\ell(\kappa) \leq m}}\left(\prod_{i=1}^{m}\left(\begin{array}{c}
n+\kappa_{i}-1 \\
\kappa_{i}
\end{array}\right)\right) \\
& \leq(2 k+1)^{m^{2}}\left(\sum_{s=0}^{2 k}\left(\begin{array}{c}
n+s-1 \\
s
\end{array}\right)\right)^{m} \\
& \leq(2 k+1)^{m^{2}}\left(\begin{array}{c}
n+2 k \\
2 k
\end{array}\right)^{m}
\end{aligned}
$$


and we obtain the announced limiting result using the classical

$$
\lim _{n / 2 k \rightarrow \rho^{-1}} \frac{1}{n} \log \left(\begin{array}{c}
n+2 k \\
2 k
\end{array}\right)=(1+\rho) \log (1+\rho)-\rho \log (\rho) .
$$

From the three-term relation (2.4), specializing to $(1, \ldots, 1)$ we get trivially

$a_{\kappa} \leq m$ and hence $\sum_{|\kappa|=k} d_{2 \kappa} a_{\kappa} \leq m \sum_{\substack{|\kappa|=2 k \\ \ell(\kappa) \leq m}} \delta_{\kappa}$ (obviously $d_{2 \kappa} \leq \delta_{2 \kappa}$ since $V_{n}^{2 \kappa}$ is contained in $F_{n}^{2 \kappa}$ ).

Then we only have to solve the equation, involving the limiting result of Theorem 5.3.

$$
s=\lim _{n / 2 k \rightarrow \rho^{-1}} \lambda_{k-1}^{(n)}=4 \frac{\rho^{-1}+1 / m}{\left(\rho^{-1}+2 / m\right)^{2}}
$$

which leads to

$$
\rho=\frac{m}{2}\left(-1+\left(1-\frac{s}{m}\right)^{-1 / 2}\right) .
$$

\section{LP VERSUS HAMMING}

In 4, A. Barg and D. Nogin give an asymptotic bound for the size of Grassmannian codes, derived from the so-called Hamming bound. They prove, with the notations of Theorem 1.1 .

Theorem 7.1. 4

$$
\frac{1}{n} \log |C| \lesssim-m \log \left(\sqrt{1-\sqrt{\frac{s+m}{2 m}}}\right)
$$

It turns out that our bound (11) is better than (21) only when $s$ is small. ${ }^{1}$ The crossing point $s_{0}$ for the two bounds has the approximate value:

\begin{tabular}{c|c|c|c|c|c}
$m$ & 2 & 3 & 4 & 5 & 6 \\
$s_{0}$ & 1.4528 & 1.2714 & 1.1853 & 1.1372 & 1.1067 \\
7 & 8 & 9 & 10 \\
1.0856 & 1.0702 & 1.0584 & 1.0492
\end{tabular}

Figure 1 plots the two bounds for $m=2$.

\section{AKNOWLEDGEMENT}

We thank Pierre de la Harpe, Claude Pache, Patrick Solé, Gregory Kabatyanskiy and Vladimir Levenshtein for helpful discussions and suggestions. Part of this work was done while the author was visiting Geneva University, supported by the Swiss National Science Foundation.

\footnotetext{
${ }^{1}$ After this paper was submitted, the authors have further improved [21), see 25].
} 


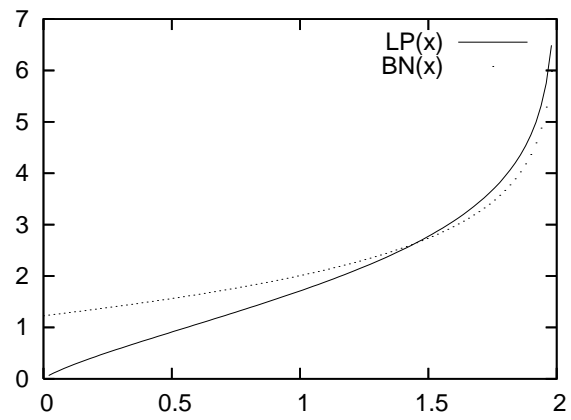

Figure 1. LP and Hamming asymptotic bounds for $m=2$

\section{REFERENCES}

[1] Ph. Delsarte, J.M. Goethals and J.J. Seidel, "Spherical codes and designs", Geom. Dedicata (6), 1977, 363-388.

[2] Ph. Delsarte, J.M. Goethals and J.J. Seidel, "Bounds for systems of lines, and Jacobi polynomials", Philips Res. Repts 30, 1975, 91-105, Bouwkamp volume.

[3] J.-H. Conway, R. H. Hardin, and N. J. A. Sloane, "Packing Lines, Planes, etc., Packings in Grassmannian Spaces", Experimental Mathematics, vol. 5, 1996, 139-159.

[4] A. Barg and D. Nogin, "Bounds on packings of spheres in the Grassmann manifold", IEEE Trans. Inform. Theory, vol. 48, no. 9, 2002, 2450-2454.

[5] C. Bachoc, E. Bannai and R. Coulangeon, "Codes and Designs in Grassmannian spaces", Discrete Mathematics, vol. 277, 2004, 15-28.

[6] C. Bachoc, R. Coulangeon and G. Nebe, "Designs in Grassmannian spaces and lattices", J. Algebraic Combinatorics vol. 16, 2002, 5-19.

[7] G. A. Kabatyanskii and V. I. Levenshtein, "Bounds for packings on a sphere and in space", Problemy Peredachi Informatsii vol. 14.1, 1978, 3-25.

[8] W. Fulton and J. Harris, Representation Theory, a first course, GTM 129, 1991, Springer.

[9] R. Goodman and N. R. Wallach, Representations and invariants of the classical groups, Encyclopedia of Mathematics and its Applications 68, 1998, Cambridge University Press.

[10] M. Lassalle, "Une formule du binôme généralisé pour les polynômes de Jack", $C . R$. Acad. Sci. Paris Sr. I Math. vol. 310 no. 5, 1990, 253-256.

[11] M. Lassalle, "Polynômes de Jacobi généralisés", C. R. Acad. Sci. Paris Sr. I Math. vol. 312 no. $6,1991,425-428$.

[12] I. G. Macdonald, Symmetric functions and Hall polynomials, 1995, Oxford Science Publications.

[13] C. Dunkl and Y. Xu, Orthogonal polynonials of several variables, Encyclopedia of Mathematics ans its Applications 81, 2001.

[14] A. T. James and A. G. Constantine, "Generalized Jacobi polynomials as spherical functions of the Grassmann manifold", Proc. London Math. Soc. vol. 29 no. 3, 1974, 174-192.

[15] G. H. Golub and C. F. van Loan, Matrix computations, 2nd edition., 1989, John Hopkins university press.

[16] T. Koornwinder, "Positivity proofs for linearization and connection coefficients of orthogonal polynomials satisfying an addition formula", J. London Math. Soc. (2), vol. 18 , no. $1,1978,101-114$. 
[17] R. J. Muirhead, Aspects of multivariate statistical theory, 1982, Wiley Series in Probability and Statistics.

[18] R. J. McEliece, E. R. Rodemich, H. Rumsey, L. Welch, "New upper bounds on the rate of a code via the Delsarte-MacWilliams inequalities", IEEE Trans. Inform. Theory IT-23, 1977, 157-166.

[19] V. I. Levenshtein, "Universal bounds for codes and designs", in Handbook of Coding Theory, eds V. Pless and W. C. Huffmann, Amsterdam: Elsevier, 1998, 499-648.

[20] A. R. Calderbank, R. H. Hardin, E. M. Rains, P. W. Shor, and N. J. A. Sloane, "A group-theoretic framework for the construction of packings in Grassmannian spaces", J. Algebraic Combin. vol. 9 no. 2, 1999, 129-140.

[21] C. Bachoc, "Designs, groups and lattices", J. Théor. Nombres Bordeaux vol. 17, 2005, 25-44.

[22] F. R. Gantmacher, The theory of matrices, vol. 2, 1960, Chelsea publishing company.

[23] L. Vretare, "Formulas for elementary spherical functions and generalized Jacobi polynomials", SIAM J. Math. Anal. vol. 15 no. 4, 1984, 805-833.

[24] M. E. H. Ismail and X. Li, "Bound on the extreme zeros of orthogonal polynomials", Proc. Amer. Math. Soc. vol. 115 no. 1, 1992, 131-140.

[25] A. Barg and D. Nogin, "A bound on Grassmannian codes", in preparation. 